\title{
1 Structures of human mGlu2 and mGlu7 homo- and heterodimers
}

2 Juan $\mathrm{Du}^{1,2,3,12}$, Dejian Wang ${ }^{1,4,12}$, Hongcheng $\mathrm{Fan}^{4,5,12}$, Chanjuan $\mathrm{Xu}^{6,12}$, Linhua Tai ${ }^{4,5,12}$,

3 Shuling Lin ${ }^{2,4,12}$, Shuo Han ${ }^{1}$, Qiuxiang $\operatorname{Tan}^{2}$, Xinwei Wang ${ }^{2,4}$, Tuo Xu ${ }^{1,4}$, Hui Zhang ${ }^{2,4}$,

4 Xiaojing $\mathrm{Chu}^{2}$, Cuiying $\mathrm{Yi}^{1}$, Peng Liu ${ }^{6}$, Xiaomei Wang ${ }^{6}$, Yu Zhou ${ }^{1,4}$, Jean-Philippe Pin ${ }^{7}$,

5 Philippe Rondard ${ }^{7}$, Hong Liu $^{1,3,4,8 *}$, Jianfeng $\operatorname{Liu}^{6,9}$, Fei Sun ${ }^{4,5,9,10} *$, Beili Wu ${ }^{2,3,4,8 *}$ \& Qiang

$$
\mathrm{Zhao}^{1,4,11 *}
$$

7

$8{ }^{1}$ State Key Laboratory of Drug Research, Shanghai Institute of Materia Medica, Chinese 9 Academy of Sciences, Shanghai, China.

$10{ }^{2}$ CAS Key Laboratory of Receptor Research, Shanghai Institute of Materia Medica, Chinese

11 Academy of Sciences, Shanghai, China.

$12{ }^{3}$ School of Pharmaceutical Science and Technology, Hangzhou Institute for Advanced Study,

13 UCAS, Hangzhou, China.

$14{ }^{4}$ University of Chinese Academy of Sciences, Beijing, China.

$15{ }^{5}$ National Laboratory of Biomacromolecules, National Center of Protein Science-Beijing,

16 CAS Center for Excellence in Biomacromolecules, Institute of Biophysics, Chinese Academy

17 of Sciences, Beijing, China

$18{ }^{6}$ Key Laboratory of Molecular Biophysics of MOE, International Research Center for

19 Sensory Biology and Technology of MOST, School of Life Science and Technology,

20 Huazhong University of Science and Technology, Wuhan, China.

$21{ }^{7}$ Institut de Génomique Fonctionnelle (IGF), Université de Montpellier, CNRS, INSERM, 2234094 Montpellier, France.

$23{ }^{8}$ School of Life Science and Technology, ShanghaiTech University, Shanghai, China.

$24{ }^{9}$ Bioland Laboratory (Guangzhou Regenerative Medicine and Health Guangdong Laboratory),

25 Guangzhou, China.

$26{ }^{10}$ Center for Biological Imaging, Institute of Biophysics, Chinese Academy of Sciences,

27 Beijing, China.

$28{ }^{11}$ Zhongshan Branch, the Institute of Drug Discovery and Development, CAS, Zhongshan, 29 China.

$30{ }^{12}$ These authors contributed equally: Juan Du, Dejian Wang, Hongcheng Fan, Chanjuan Xu, 31 Linhua Tai, Shuling Lin.

$32 *$ *-mail: hliu@simm.ac.cn; jfliu@mail.hust.edu.cn; feisun@ibp.ac.cn; beiliwu@simm.ac.cn;

33 zhaoq@simm.ac.cn. 
35 The metabotropic glutamate receptors (mGlus) are involved in modulation of synaptic

36 transmission and neuronal excitability in the central nervous system ${ }^{1}$. These receptors

37 likely exist as both homo- and heterodimers with unique pharmacological and

38 functional properties ${ }^{2-4}$. Here we report four cryo-electron microscopy structures of the

39 human mGlus, including inactive mGlu2 and mGlu7 homodimers, agonist/PAM-bound

40 mGlu2 homodimer, and inactive mGlu2-7 heterodimer. A subtype-dependent

41 dimerization mode of mGlus was observed, as a unique dimer interface mediated by

42 helix IV important to limit receptor activity exists in the inactive mGlu2 structure only.

43 The structures provide molecular details of inter- and intrasubunit conformational

44 changes that are required for receptor activation, which distinguish class C G-protein-

45 coupled receptors (GPCRs) from classes A and B receptors. Furthermore, the mGlu2-7

46 heterodimer structure and functional studies suggest that the mGlu7 subunit plays a

47 dominant role in controlling dimeric association and $\mathbf{G}$ protein activation in the

48 heterodimer. These insights into mGlu homo- and heterodimers highlight the complex

49 landscape of mGlu dimerization and activation.

50 The mGlus contain a large extracellular domain (ECD) composed of the Venus flytrap (VFT)

51 domain that binds agonist and a cysteine-rich domain (CRD) connected to a seven-helical

52 transmembrane domain (TMD) responsible for $\mathrm{G}$ protein coupling ${ }^{4}$. It has been

53 acknowledged that homodimerization is mandatory for the function of the mGlus, making

54 them complex allosteric proteins with two subunits influencing each other ${ }^{3}$. In addition, there

55 is increasing evidence suggesting that different mGlu subunits can associate to form multiple

56 types of heterodimers, adding complexity of function modulation in this receptor family ${ }^{2,4}$.

57 However, conformational difference between the mGlu homo- and heterodimerization and

58 how these dimers control mGlu function remain unclear. Thus, we performed single-particle 
cryo-electron microscopy (cryo-EM), X-ray crystallography, and functional studies to solve

60 structures of mGlu2 and mGlu7 in both homo- and heterodimeric organizations and provide

61 valuable insights into mGlu activity modulation.

\section{Structure determination of mGlu2 and mGlu7}

63 For structure determination of inactive mGlu2 and mGlu7 homodimers, a modified mGlu2 or

64 mGlu7 was purified in presence of its selective negative allosteric modulator (NAM)

65 [NAM563 (ref. 5) for mGlu2; MMPIP ${ }^{6}$ for mGlu7] (see Methods for construct design and

66 function characterization; Extended Data Fig. 1a-1). The stable agonist-bound mGlu2 was

67 obtained by co-purifying another modified receptor with the agonist LY354740 (ref. 7), the

68 positive allosteric modulator (PAM) JNJ-40411813 (ref. 8), and the nanobody DN13 that acts

69 as an mGlu2 PAM $^{9}$ (Extended Data Fig. 1e, i, n-q). To solve the mGlu2-7 heterodimer

70 structure, an FK506 binding protein (FKBP)-tagged mGlu2 and a rapamycin binding

71 fragment (FRB)-tagged mGlu7 were co-purified in the presence of NAM563 and MMPIP

72 (Extended Data Fig. 1r and Supplementary Fig. 1). The inactive structures of mGlu2, mGlu7,

73 and mGlu2-7 and the agonist/PAM-bound mGlu2 structure were determined by cryo-EM

74 with overall resolutions of $3.6 \AA, 4.0 \AA, 3.9 \AA$, and $3.1 \AA$, respectively, and the resolutions in

75 the ECDs of mGlu7 and mGlu2-7 were further improved to $3.6 \AA$ and $3.5 \AA$, respectively

76 (Extended Data Figs. 2 and 3; Extended Data Table 1). To provide high-resolution molecular

77 details of NAM binding mode, we also solved two crystal structures of the mGlu2 TMD

78 bound to two NAMs, NAM563 and NAM597 (ref. 10), at $2.5 \AA$ and $2.7 \AA$ resolution,

79 respectively (Extended Data Table 2).

80 The inter-VFT disulfide bonds (mGlu2, C121-C121; mGlu7, C136-C136; mGlu2-7,

81 C121-C136) were resolved in all the structures, but exhibit distinct conformations (Extended

82 Data Fig. 3). Compared to the inactive mGlu2 structure, the junction loop between the 
83 disulfide bond and B helix in the VFT adopts a more extended conformation in the inactive 84 structures of mGlu7 and mGlu2-7, resulting in a higher position of this disulfide bond

85 (Extended Data Fig. 4a). Associated with the conformational rearrangement of the VFTs 86 upon agonist binding, the disulfide bond undergoes an upward movement in the 87 agonist/PAM-bound mGlu2 structure relative to the inactive state (Extended Data Fig. 4b), 88 which may be consistent with previous observation that the intersubunit disulfide bond 89 stabilizes the receptor active state ${ }^{2}$.

\section{Multiple dimerization modes of inactive mGlus}

91 Similar to the previously published inactive structures of mGlu5 ${ }^{11}$ and mGlu2-VFT ${ }^{12}$, the

92 inactive mGlu2 and mGlu7 structures reveal an open conformation of the VFTs and a 93 common homodimer interface at the apex of the VFTs (Extended Data Fig. 4c, d). In contrast, 94 the CRDs adopt distinct relative positioning in the inactive structures of mGlu2, mGlu5, and 95 mGlu7, with the CRDs in mGlu2 exhibiting the closest positions (Extended Data Fig. 4e).

96 This difference is associated with the distinct dimerization modes of the receptor TMDs, 97 where close contact is observed in mGlu2 but is absent in mGlu5 and mGlu7 (Fig. 2a). The 98 TMDs of mGlu5 and mGlu7 are completely separated with helices $\mathrm{V}$ in the two subunits

99 facing each other, while the TMDs in the inactive mGlu2 form extensive interactions along 100 the whole length of helix IV (Figs. 1a, b and 2a). This dimerization mode is supported by 101 previous crosslinking studies, showing that helices IV and V of mGlu2 are involved in 102 intersubunit interaction in basal or inactive state ${ }^{13}$. The additional involvement of helix $\mathrm{V}$ in 103 dimerization suggests a dynamic nature of the dimer interface.

104 The different dimerization modes of the inactive mGlus agree with previous investigation 105 of inter-TMD interaction, in which the mGlu2 TMD showed a higher proportion of dimers 106 compared to that of mGlu1, mGlu3, or mGlu $5^{14}$, suggesting subtype-specific inter-TMD 
107 interaction strength of mGlus. In the inactive mGlu2, the TMD dimer interface is mainly

108 mediated by helix IV and the extracellular tip of helix III through hydrophobic contacts (Fig.

$1092 \mathrm{~b}, \mathrm{c})$. Mutations of most of the key residues in the dimer interface resulted in an elevated

110 constitutive activity (Fig. 2d and Extended Data Table 3), and the full dose-response curves

111 excluded the possible effects of increased glutamate potency and maximal activation

112 potentially induced by the environmental glutamate (Extended Data Fig. 1s and Extended

113 Data Table 3). Furthermore, a substantially impaired receptor activity was observed when the

114 inactive dimer interface is locked through a disulfide bridge by introducing a cysteine

115 substitution, L698 ${ }^{4.51} \mathrm{C}$ or V699 ${ }^{4.52} \mathrm{C}$ (Extended Data Fig. 1u, v and Supplementary Fig. 1).

116 These data imply that the helix IV-mediated dimerization plays a role in stabilizing the

117 receptor inactive conformation.

118 The mGlu2 residues that participate in dimerization are poorly conserved between mGlus

119 (Extended Data Fig. 5), suggesting that the mGlu2 dimer interface along helix IV may be 120 subtype-dependent. Some of the key residues such as L684 ${ }^{4.37}$, L692 $2^{4.45}$, L698 ${ }^{4.51}$, and $121 \mathrm{~V} 699^{4.52}$ are substituted by phenylalanine or residues with small side chains in other mGlus, 122 which may disturb the dimer interface by introducing a spatial hindrance or disrupting the 123 hydrophobic interaction. Replacing these residues with their counterparts in other mGlus 124 increased the receptor basal activity (Fig. 2d, Extended Data Fig. 1t, and Extended Data 125 Table 3), further reflecting the disturbance of the inactive state. In contrast, a wild-type level 126 of basal activity was observed for the mGlu7 mutants in helices III and IV (Fig. 2e). These 127 data support that the dimer interface along helix IV in the inactive mGlu2 homodimer is 128 subtype-specific. However, this does not rule out the possibilities of specific inactive 129 conformation that is required for facilitating conformational change upon receptor activation 130 and structural constraints coming from the specific conformation of the VFTs and CRDs in 131 different mGlus. 


\section{Intersubunit interactions upon activation}

133 Comparison of the inactive and agonist/PAM-bound structures of mGlu2 revealed a closure

134 of the VFTs and CRDs upon receptor activation (Fig. 1b, c; Extended Data Fig. 4f, g),

135 leading to a dimer interface mediated by a hydrophobic interaction core at the apex of the

136 CRDs (Extended Data Fig. 4h). Locking this interface by introducing a disulfide bond

137 resulted in constitutive activation of mGlu2 and mGlu7 (Extended Data Fig. 1w, x),

138 consistent with previous studies showing that a precise association of the two CRDs in an

139 mGlu dimer is required for full receptor activation ${ }^{15}$.

140 Similar to what was observed for mGlu5 ${ }^{11}$, in the agonist/PAM-bound mGlu2 structure

141 the CRD and ECL2 are in proximity (Extended Data Fig. 6a and Extended Data Fig. 3c).

142 Disturbing the interaction interface by introducing alanine mutation(s) or removing the ECL2

143 residues R714 and E715 substantially reduced the maximal response $\left(E_{\max }\right)$ of the receptor in

144 the agonist-induced inositol phosphate (IP) accumulation assay but had no effect on the

145 PAM-induced receptor signalling (Extended Data Fig. 1y, z; Extended Data Table 3). A

146 similar detrimental effect was also shown when residues at the apex of ECL2 in mGlu7

147 (T737-E741) were removed (Extended Data Fig. 1x and Extended Data Table 3). These data

148 suggest that the CRD and ECL2 may play a similar role in transmitting signal from the VFT

149 to the TMD in different mGlus.

150 Upon receptor activation, the helix IV-mediated dimeric association of mGlu2 switches to

151 an active state mainly contributed by helix VI (Fig. 1b, c), a hallmark of class C GPCR

152 activation $^{11,13}$. An increased constitutive activity of mGlu7 mutant $\mathrm{A} 812^{6.61} \mathrm{C}$ was observed

153 (Extended Data Fig. 1x), suggesting that helix VI is most likely also involved in mGlu7

154 dimerization in the active state. Despite a similar dimer interface along helix VI in mGlu2

155 and mGlu5, the agonist/PAM-bound mGlu2 structure reveals a slight movement of the TMDs 
156 relative to the mGlu5 structure, leading to additional contacts between residues $\mathrm{V} 782^{6.59}$ and

157 V789 $9^{7.27}$ at the extracellular tips of helices VI and VII (Extended Data Fig. 6b). Our cysteine

158 crosslinking and signalling studies showed that in contrast to mutant $\mathrm{C} 121 \mathrm{~A}$, additional

159 introduction of mutations $\mathrm{V} 782^{6.59} \mathrm{C}$ and $\mathrm{V} 789^{7.27} \mathrm{C}$ in the two subunits, respectively,

160 displayed an increased dimer proportion in presence of agonist and PAM (Extended Data Fig.

161 1u and Supplementary Fig. 1) and an elevated constitutive activity (Extended Data Table 3),

162 which reflect the close contact between helices VI and VII in the active state and importance

163 of such contact in stabilizing the receptor active conformation.

\section{Intrasubunit conformational change of mGlu2}

165 Following the relative movement of the two subunits, further conformational change within

166 the TMD of one subunit occurs, resulting in G protein activation ${ }^{16}$. The crystal structures of

167 the mGlu2-TMD bound to NAM563 and NAM597 (Extended Data Fig. 6c) and our recently

168 determined structure of the fully active $m G l u 2-\mathrm{G}_{\mathrm{i}}$ complex ${ }^{17}$ allowed us to obtain molecular

169 details of the conformational changes required for receptor activation within the receptor

170 TMD.

In mGlu2, NAM563 and NAM597 bind to a cavity mainly formed by helices III and V-

172 VII, which is similar to the NAM-binding pockets previously observed in mGlu1 ${ }^{18}$ and 173 mGlu $^{19}$ and the ligand-binding sites in some class A GPCRs (Extended Data Fig. 6d). These 174 two NAMs exhibit similar binding patterns that share many common interactions with the 175 receptor but are distinct on the extracellular side, with the trifluoromethyl-morpholin group of 176 NAM563 squeezing into a gap between helices VI and VII while the methyl-pyrazol group in 177 NAM597 extending in an opposite direction to enable extra interactions with helix II and 178 ECL2 (Extended Data Fig. 6c). This may account for the slightly higher activity of NAM597 179 in inhibiting receptor signalling (Extended Data Fig. 1m and Extended Data Table 3). The 
180 binding modes of these two NAMs are supported by a 5-1,000-fold reduction of their 181 inhibitory activity associated with mutations within the binding pocket, which had a weak 182 effect on agonist-induced receptor signalling (Extended Data Table 3).

183 Compared to the inactive structures of mGlu2 and mGlu2-TMD-NAMs, the pronounced 184 outward movement of helix VI, a hallmark of receptor activation for classes A and B GPCRs, 185 was not observed in both the $\mathrm{G}_{\mathrm{i}}$-bound and -free structures of agonist-bound mGlu2 186 (Extended Data Fig. 6e). Instead, helix VI in the $\mathrm{G}_{\mathrm{i}}$-bound subunit of the $\mathrm{mGlu}_{2}-\mathrm{G}_{\mathrm{i}}$ complex 187 undergoes a downward shift towards the intracellular surface by half a helical turn (Extended 188 Data Fig. 6f). However, neither the $G$ protein-free agonist/PAM-bound structure nor the $G_{i^{-}}$ 189 free subunit in the $m$ Glu2 $-\mathrm{G}_{\mathrm{i}}$ structure displays such conformational change, suggesting that 190 this movement is associated with G protein binding. Close inspection of the mGlu2-TMD191 NAM and mGlu2- $\mathrm{G}_{\mathrm{i}}$ structures revealed a rotamer conformational change of the highly 192 conserved class C GPCR residue $\mathrm{W}^{6.50}$ with its side chain rotating downwards by 193 approximate $90^{\circ}$ in the $\mathrm{G}_{\mathrm{i}}$-bound structure, which may release the transmembrane helical 194 bundle from its inactive state by disrupting an interaction core involving helices III and V195 VII and potentially leads to the downward shift of helix VI (Extended Data Fig. 6g). In the 196 structures of mGlu2-TMD-NAMs, the NAMs form a close contact with W773 $3^{6.50}$, 197 constraining the conformational change of the side chain to stabilize the receptor in the inactive state (Extended Data Fig. 6c).

199 Although not directly involved in $\mathrm{G}$ protein binding of $\mathrm{mGlu} 2^{17}$, helix VI may play a role 200 in modulating receptor activation by altering the interaction network on the intracellular 201 surface of the receptor to facilitate $\mathrm{G}$ protein recognition. A salt bridge between the highly 202 conserved residues $\mathrm{K}^{3.50}$ and $\mathrm{E}^{6.35}$, which is known as the 'ionic lock' of class C GPCRs ${ }^{18,19}$, 203 and another ionic interaction formed by the less well conserved residue R656 $6^{3.53}$ in helix III 204 and E754 in ICL3 are intact in both mGlu2-TMD-NAM structures but broken in the $\mathrm{G}_{\mathrm{i}^{-}}$ 
205 bound subunit of the mGlu2- $\mathrm{G}_{\mathrm{i}}$ complex likely due to the downward shift of helix VI and 206 rearrangement of ICL3 (Extended Data Fig. 6h). Our signalling assay showed an increased 207 constitutive activity resulted from mutation E754A (Extended Data Table 3), implying that

208 the salt bridge between R656 $6^{3.53}$ and E754 may act as a secondary 'ionic lock' to stabilize the 209 inactive conformation of mGlu2.

\section{Heterodimerization of mGlu2 and mGlu7}

211 It was recently found that mGlu2 can associate with mGlu7 in hippocampus, suggesting a 212 physiological relevance of such a heterodimer ${ }^{20}$. The mGlu2-7 heterodimerization was further 213 confirmed by our tr-fluorescence resonance energy transfer (tr-FRET) assay (Extended Data 214 Fig. 7a, b). Like the homodimers, the VFTs in the mGlu2-7 heterodimer adopt a similar open 215 conformation forming contacts mainly through the B and C helices (Extended Data Fig. 4i, j), 216 suggesting a conserved VFT dimerization mode shared by the mGlu homo- and heterodimers.

217 In addition to this common dimer interface, extra contacts between the mGlu7 residue R191 218 and the mGlu2 residues D174 and S176 were observed in the heterodimer (Fig. 3a). Similar 219 interactions also exist in the inactive mGlu2 homodimer, but not in the inactive mGlu7 220 homodimer due to the substitution of S176 (mGlu2) with R190 (mGlu7), which repels the 221 residue R191 in the opposite subunit (Fig. 3a). Compared to the heterodimer mutant that 222 lacks the inter-VFT disulfide bond, further introduction of mutation D174C or S176C in 223 mGlu2 and mutation R191C in mGlu7 greatly increased the dimer proportion in our 224 crosslinking studies (Extended Data Fig. 1aa and Supplementary Fig. 1), supporting the close 225 contacts between these residues. A substantially reduced potency of both the mGlu2 and 226 mGlu7 agonists was observed for the crosslinked heterodimer mutants and mutants with the 227 mGlu2 residues D174 and S176 replaced with arginines or alanine mutation(s) introduced to 228 disrupt the inter-VFT interactions (Extended Data Fig. 7c-g), suggesting that this heterodimer 229 interface may play a role in modulating receptor activation. 
230 The interaction difference in the top lobes of the VFTs brings the rest of the heterodimer 231 in closer distance compared to that in the mGlu7 homodimer (Fig. 3a). While the TMDs in 232 the mGlu2 and mGlu7 homodimers adopt distinct dimerization modes, the TMDs in the 233 mGlu2-7 heterodimer assemble in a similar manner to that in the mGlu7 homodimer with 234 helices $\mathrm{V}$ facing each other (Fig. 1d), implying that mGlu7 may be more dominant in 235 controlling heterodimer assembly. Previous investigations of mGlu homo- and 236 heterodimerization suggested that mGlu2 dimerized with mGlu7 with a lower propensity 237 compared to that of mGlu2 homodimerization ${ }^{2}$. This is likely due to the distinct dimerization 238 modes of the TMDs, which form extensive contacts in the mGlu2 homodimer but not in the 239 mGlu2-7 heterodimer.

240 Asymmetric activation has been observed for some mGlu heterodimers ${ }^{2,21}$. The mGlu2-7 heterodimer displayed a 3 -fold higher $E_{\max }$ than the mGlu7 homodimer when activated by the mGlu7 agonist L-AP4 (ref. 22) (Fig. 3c), which indicates that the heterodimerization boosts the $G$ protein activation even when one of the subunits is not occupied with agonist, 244 demonstrating a clear cooperativity between the two subunits. Further investigation of 245 heterodimer signal transduction showed an agonist-induced response comparable to the 246 controls when the mGlu2 subunit carried mutation F756S in ICL3 that blocks the G protein 247 coupling ${ }^{23}$, while no response was observed if the mutation was introduced in the mGlu7 248 subunit (Fig. 3b, c). Such data imply that the mGlu7 subunit is mainly responsible for G 249 protein activation within the mGlu2-7 heterodimer, consistent with previous observation that 250 the group III mGlu subunit plays a predominant role in mediating signal transduction in the 251 heterodimers between groups II and III mGlus ${ }^{21}$. Furthermore, a recovery of agonist-induced 252 response was observed in presence of the mGlu2 PAM JNJ-40411813 with the heterodimer 253 containing the blocked mGlu7 subunit (Fig. 3d, e), demonstrating that the mGlu2 subunit 254 within the mGlu2-7 heterodimer is capable of G protein coupling when its TMD activation is 
257 Extended Data Fig. 7h). This is in contrast to the previous finding on the mGlu2-4

258 heterodimer, in which mGlu2 took over for G protein activation when an mGlu4 NAM was

259 used $^{21}$. These observations suggest subtype-specific manners of signal transduction within

260 different mGlu heterodimers.

\section{References}

2621 Niswender, C. M. \& Conn, P. J. Metabotropic glutamate receptors: physiology, pharmacology, and disease. Annu. Rev. Pharmacol. Toxicol. 50, 295-322 (2010).

2 Levitz, J. et al. Mechanism of assembly and cooperativity of homomeric and heteromeric metabotropic glutamate receptors. Neuron 92, 143-159 (2016).

3 Kniazeff, J., Prezeau, L., Rondard, P., Pin, J. P. \& Goudet, C. Dimers and beyond: The functional puzzles of class C GPCRs. Pharmacol. Ther. 130, 9-25 (2011).

4 Pin, J. P. \& Bettler, B. Organization and functions of mGlu and $\mathrm{GABA}_{\mathrm{B}}$ receptor complexes. Nature 540, 60-68 (2016).

5 Bungard, C. J. et al. Quinoline carboxamide and quinoline carbonitrile derivatives as mGluR2-negative allosteric modulators, compositions, and their use. PCT Int. Pat. Appl WO 2013/066736 A1 (2013).

6 Suzuki, G. et al. In vitro pharmacological characterization of novel isoxazolopyridone derivatives as allosteric metabotropic glutamate receptor 7 antagonists. J. Pharmacol. Exp. Ther. 323, 147-156 (2007).

7 Monn, J. A. et al. Design, synthesis, and pharmacological characterization of (+)-2aminobicyclo[3.1.0]hexane-2,6-dicarboxylic acid (LY354740): a potent, selective, and orally active group 2 metabotropic glutamate receptor agonist possessing anticonvulsant and anxiolytic properties. J. Med. Chem. 40, 528-537 (1997).

8 Cid, J. M. et al. Discovery of 1-butyl-3-chloro-4-(4-phenyl-1-piperidinyl)-(1H)-pyridone (JNJ-40411813): a novel positive allosteric modulator of the metabotropic glutamate 2 receptor. J. Med. Chem. 57, 6495-6512 (2014).

9 Scholler, P. et al. Allosteric nanobodies uncover a role of hippocampal mGlu2 receptor homodimers in contextual fear consolidation. Nat. Commun. 8, 1967 (2017).

10 Arasappan, A. et al. Tetrahydronaphthyridine derivatives as mGluR2-negative allosteric modulators, compositions, and their use. PCT Int. Pat. Appl WO 2016/029454 A1 (2016).

11 Koehl, A. et al. Structural insights into the activation of metabotropic glutamate receptors. Nature 566, 79-84 (2019).

12 Chappell, M. D. et al. Discovery of $(1 S, 2 R, 3 S, 4 S, 5 R, 6 R)-2$-Amino-3-[(3,4difluorophenyl)sulfanylmethyl]-4-hydroxy-bicy clo[3.1.0]hexane-2,6-dicarboxylic Acid Hydrochloride (LY3020371.HCl): A Potent, Metabotropic Glutamate 2/3 Receptor Antagonist with Antidepressant-Like Activity. J. Med. Chem. 59, 10974-10993 (2016).

13 Xue, L. et al. Major ligand-induced rearrangement of the heptahelical domain interface in a GPCR dimer. Nat. Chem. Biol. 11, 134-140 (2015).

14 Gutzeit, V. A. et al. Conformational dynamics between transmembrane domains and allosteric modulation of a metabotropic glutamate receptor. Elife 8 (2019).

15 Huang, S. et al. Interdomain movements in metabotropic glutamate receptor activation. Proc. Natl. Acad. Sci. U S A 108, 15480-15485 (2011). 
16 Hlavackova, V. et al. Sequential inter- and intrasubunit rearrangements during activation of dimeric metabotropic glutamate receptor 1. Sci. Signal. 5, ra59 (2012).

30117 Lin, S. et al. Structures of $\mathrm{G}_{\mathrm{i}}$-bound metabotropic glutamate receptors mGlu2 and mGlu4.

$18 \mathrm{Wu}, \mathrm{H}$. et al. Structure of a class C GPCR metabotropic glutamate receptor 1 bound to an allosteric modulator. Science 344, 58-64 (2014).

19 Dore, A. S. et al. Structure of class C GPCR metabotropic glutamate receptor 5 transmembrane domain. Nature 511, 557-562 (2014).

20 Habrian, C. H. et al. Conformational pathway provides unique sensitivity to a synaptic mGluR. Nat. Commun. 10, 5572 (2019).

21 Liu, J. et al. Allosteric control of an asymmetric transduction in a $\mathrm{G}$ protein-coupled receptor heterodimer. Elife 6 (2017).

22 Saugstad, J. A., Kinzie, J. M., Mulvihill, E. R., Segerson, T. P. \& Westbrook, G. L. Cloning and expression of a new member of the L-2-amino-4-phosphonobutyric acid-sensitive class of metabotropic glutamate receptors. Mol. Pharmacol. 45, 367-372 (1994).

23 Francesconi, A. \& Duvoisin, R. M. Role of the second and third intracellular loops of metabotropic glutamate receptors in mediating dual signal transduction activation. J. Biol. Chem. 273, 5615-5624 (1998). 


\section{Figure legends}

319 Fig. 1 I Maps and structures of mGlu2 and mGlu7. The maps and structures are colored

320 according to chains. The structures are shown in both side and intracellular views. a, Inactive

321 mGlu7 homodimer. The red arrow indicates the most proximal transmembrane helix pair, 322 with the distance between the intracellular tips labelled. The disulfide bonds are shown as

323 yellow sticks. b, Inactive mGlu2 homodimer. c, Agonist/PAM-bound mGlu2 homodimer. d,

324 Inactive mGlu2-7 heterodimer.

325 Fig. 2 I Inactive mGlu structures. a, Comparison of the TMDs in the inactive structures of

326 mGlu2, mGlu5 (PDB ID: 6N52), and mGlu7. The distance between the C $\alpha$ atoms of the

327 residues at position 5.41 in each structure is indicated by a dashed line. The red arrow 328 indicates the movement of the TMDs in mGlu5 and mGlu7 relative to that in mGlu2. b, c,

329 The TMD dimer interface in the inactive mGlu2 structure. The residues that are involved in 330 dimerization are shown as sticks. d, Basal activity of the wild-type (WT) mGlu2 and mutants 331 measured by IP accumulation assay. The basal activity of the WT was substantially reduced 332 by the orthosteric antagonist LY341495 $(50 \mu \mathrm{M})$ or the NAM NAM563 $(25 \mu \mathrm{M})$. e, Basal 333 activity of the WT mGlu7 and mutants. The basal activity of the WT was substantially 334 reduced by the orthosteric antagonist XAP044 $(25 \mu \mathrm{M})$ or the NAM MMPIP $(25 \mu \mathrm{M})$. Data 335 are presented as percent of WT activity and displayed as mean \pm SEM (bars) from at least 336 five independent experiments performed in technical triplicate with individual data points 337 shown (dots). $* * * P<0.001$ by one-way ANOVA followed by Dunnett's post-test compared to 338 the response of WT. See Extended Data Table 3 for detailed independent experiment 339 numbers (n), $P$ values, and expression level.

340 Fig. 3 I Structure and activation of mGlu2-7 heterodimer. a, Structural comparison of 341 inactive mGlu2-7 heterodimer and mGlu7 homodimer. The distances between the conserved 
342 cysteine residues (C540 in mGlu2, C563 in mGlu7) in the two structures are shown as dashed 343 lines (right middle panel). The red arrows indicate the movement of each transmembrane 344 helix in mGlu2-7 relative to mGlu7 (right bottom panel). b-e, Calcium release assays of 345 mGlu2 and mGlu7 homo- and heterodimers. Data are shown as mean \pm SEM from at least 346 three independent experiments performed in technical triplicate and correspond to the peak 347 response. The numbers of independent experiments (n) are shown in the parentheses. 348 Schemes illustrating the activation of the mGlu2-7 heterodimer upon binding to agonist alone 349 or agonist together with mGlu2 PAM (or mGlu7 NAM) are shown on the right of each panel. 350 b, LY354740-induced calcium release of mGlu2 and mGlu2-7. ' $x$ ' indicates the $\mathrm{F}^{\mathrm{ICL} 3} \mathrm{~S}$ 351 mutation introduced in the mGlu2 or mGlu7 subunit. c, L-AP4-induced calcium release of 352 mGlu7 and mGlu2-7. d, Calcium release of mGlu2-7 $7^{\times}$in presence of LY354740 or 353 LY354740+mGlu2 PAM (JNJ-40411813)/mGlu7 NAM (MMPIP, ADX71743 or MDIP). e, 354 Calcium release of mGlu2-7 ${ }^{\times}$in presence of L-AP4 or L-AP4+JNJ-40411813. 
356 Construct design and expression of mGlu2, mGlu7, and mGlu2-7

357 To solve the inactive mGlu2 structure, the human mGlu2 gene was cloned into a modified 358 pTT5 vector with a hemagglutinin (HA) signal peptide and a Flag-tag at the $\mathrm{N}$ terminus and 359 a PreScission protease (PPase) site and a $10 \times$ His-tag at the $\mathrm{C}$ terminus. For the inactive 360 mGlu7, the human mGlu7 gene was cloned into the same vector with the HA signal peptide 361 at the $\mathrm{N}$ terminus and the PPase site, $2 \times$ Strep-tag, and Flag-tag at the $\mathrm{C}$ terminus. The 362 flexible $\mathrm{C}$ termini of the two receptors were truncated with different lengths, among which 363 removing 47 residues (V826-L872) in mGlu2 and 56 residues (K860-I915) in mGlu7 364 produced the most optimal protein yield and homogeneity (Extended Data Fig. 1a, b). To 365 further improve stability of the inactive receptors, mutation $\mathrm{N}^{3.52} \mathrm{Y}$ was introduced in both 366 receptors as suggested by previous structural studies of mGlu5-TMD ${ }^{19}$. Furthermore, 367 mutation $\mathrm{H} 815^{7.53} \mathrm{Y}$ in mGlu2 and mutations $\mathrm{G} 722^{4.52} \mathrm{I}, \mathrm{I} 775^{5.59} \mathrm{~F}$, and $\mathrm{P} 789^{6.38} \mathrm{Y}$ in mGlu7

368 were designed by replacing these residues with their counterparts in other mGlus to 369 potentially increase stability of the transmembrane helical bundle by introducing extra intra370 and/or inter-helical contacts through larger side chains (Extended Data Fig. 5). With these 371 mutations, the protein yield and homogeneity were greatly improved (Extended Data Fig. 1c, 372 d). To assess effects of the truncations and mutations on receptor function, we performed IP 373 accumulation assay and a bioluminescence resonance energy transfer (BRET) assay using

374 TRUPATH biosensors ${ }^{24}$, which measures the proximal interaction between the $\alpha$ and $\gamma$ 375 subunits upon $\mathrm{G}_{\mathrm{i}}$ activation. The results confirmed that these modifications had little effect on 376 receptor signalling and NAM activity (Extended Data Fig. 1e-j).

377 For structural studies of agonist-bound mGlu2, the wild-type receptor was modified by 378 introducing a mutation S601A, which, together with the C-terminal truncation (residues 
379 V826-L872), greatly improved protein yield (Extended Data Fig. 1n) but did not alter

380 receptor activation and PAM activity (Extended Data Fig. 1e, i, o, p). In addition, the C-

381 terminal His-tag was replaced by a $2 \times$ Strep-tag to facilitate protein purification.

382 To aid formation of mGlu2-7 heterodimer, two fusion proteins FKBP and FRB, which

383 form an interaction pair to stabilize the heterodimerization ${ }^{25}$, were linked to the $\mathrm{C}$ termini of 384 mGlu2 (E19-V825) and mGlu7 (Q35-R859), respectively. FRET analysis indicates that these 385 fusions do not affect heterodimerization between these two mGlus (Extended Data Fig. 7a, b). 386 To allow tandem affinity-chromatography, $10 \times$ His-tag and $2 \times$ Strep-tag were added to the $387 \mathrm{C}$ terminus of mGlu2-FKBP and the $\mathrm{N}$ terminus of mGlu7-FRB, respectively. The mGlu2 388 mutations $\mathrm{N} 655^{3.52} \mathrm{Y}$ and $\mathrm{H} 815^{7.53} \mathrm{Y}$ and the mGlu7 mutations $\mathrm{G} 722^{4.52} \mathrm{I}$, I775 ${ }^{5.59} \mathrm{~F}$, and $389 \mathrm{P} 789^{6.38} \mathrm{Y}$ were introduced to facilitate expression and purification of the heterodimer.

390 All the constructs were expressed in HEK293F cells (Invitrogen) with the starting density 391 at $1.2 \times 10^{6}$ cells $\mathrm{ml}^{-1}$ in $5 \% \mathrm{CO}_{2}$ at $37^{\circ} \mathrm{C}$. For the mGlu2-7 heterodimer, the mGlu2 and 392 mGlu7 constructs were co-expressed with a plasmid ratio of 1:1 and $100 \mathrm{nM}$ rapamycin was 393 added during expression. The cells were harvested by centrifugation (1,000g for $15 \mathrm{~min})$ after $39448 \mathrm{~h}$ transfection and stored at $-80^{\circ} \mathrm{C}$ until use.

\section{Purification of mGlu2 and mGlu7 homo- and heterodimers}

396 The cells expressing mGlu2, mGlu7, or mGlu2-7 were lysed in a hypotonic buffer containing $39710 \mathrm{mM}$ HEPES, pH 7.5, $10 \mathrm{mM} \mathrm{MgCl}, 20 \mathrm{mM} \mathrm{KCl}$, and EDTA-free protease inhibitor 398 cocktail tablets (Roche). Extensive washes of membranes were performed by repeated 399 dounce homogenization and centrifugation (160,000 $\mathrm{g}$ for $30 \mathrm{~min})$ in the hypotonic buffer and 400 a high-osmotic buffer (hypotonic buffer supplemented with $1 \mathrm{M} \mathrm{NaCl}$ ). The purified 401 membranes were suspended in $10 \mathrm{mM}$ HEPES, pH 7.5, $10 \mathrm{mM} \mathrm{MgCl} 2,20 \mathrm{mM} \mathrm{KCl}$, and 30\% 402 glycerol and stored at $-80^{\circ} \mathrm{C}$ until use. 
403 The membranes were thawed and incubated with different ligands [50 $\mu$ M NAM563 for 404 inactive mGlu2; $50 \mu \mathrm{M}$ MMPIP for inactive mGlu7; $50 \mu \mathrm{M}$ LY354740, $50 \mu \mathrm{M}$ JNJ40540411813 , and DN13 (prepared as below) (DN13 : mGlu2 = 3:1) for agonist/PAM-bound 406 mGlu2; $50 \mu \mathrm{M}$ NAM563 and $25 \mu \mathrm{M}$ MMPIP for mGlu2-7 heterodimer], $1 \mathrm{mg} \mathrm{ml}^{-1}$ 407 iodoacetamide (Sigma), and EDTA-free protease inhibitor cocktail tablets at $4{ }^{\circ} \mathrm{C}$ for $1 \mathrm{~h}$. 408 After that, equal volume of solubilization buffer containing $100 \mathrm{mM}$ HEPES, $\mathrm{pH}$ 7.5, 300 $409 \mathrm{mM} \mathrm{NaCl}, 1 \%(\mathrm{w} / \mathrm{v})$ n-dodecyl- $\beta$-D-maltopyranoside (DDM, Anatrace) and 0.2\% (w/v) 410 cholesteryl hemisuccinate (CHS, Sigma) was added to the membranes and incubated at $4{ }^{\circ} \mathrm{C}$ 411 for $3 \mathrm{~h}$. The supernatant was then isolated by centrifugation at $160,000 \mathrm{~g}$ for $30 \mathrm{~min}$, and 412 incubated with TALON resin (Clontech) (for the inactive mGlu2 and mGlu2-7) or STREP 413 resin (IBA) (for the agonist/PAM-bound mGlu2 and inactive mGlu7) at $4{ }^{\circ} \mathrm{C}$ overnight.

414 To purify the inactive mGlu2 homodimer, the TALON resin was washed with 10 column 415 volumes of $0.05 \%$ (w/v) DDM, 0.01\% (w/v) CHS, $25 \mathrm{mM}$ HEPES, $\mathrm{pH}$ 7.5, $150 \mathrm{mM} \mathrm{NaCl}$, $41630 \mathrm{mM}$ imidazole, and $50 \mu \mathrm{M}$ NAM563. Then the detergent was exchanged to glyco417 diosgenin (GDN, Anatrace) by incubating the resin with a buffer containing $50 \mathrm{mM}$ HEPES, $418 \mathrm{pH} 7.5,0.25 \%(\mathrm{w} / \mathrm{v}) \mathrm{GDN}, 150 \mathrm{mM} \mathrm{NaCl}, 30 \mathrm{mM}$ imidazole, and $50 \mu \mathrm{M}$ NAM563 at $4{ }^{\circ} \mathrm{C}$ 419 for $2 \mathrm{~h}$. The resin was then washed by another 10 column volumes of wash buffer containing $42025 \mathrm{mM}$ HEPES, pH 7.5, 0.01\% (w/v) GDN, $150 \mathrm{mM} \mathrm{NaCl}, 30 \mathrm{mM}$ imidazole, and $50 \mu \mathrm{M}$ 421 NAM563. The protein was then eluted by the wash buffer supplemented with $300 \mathrm{mM}$ 422 imidazole. The imidazole was removed using a PD MiniTrap G-25 column (GE healthcare), 423 and the protein was treated with His-tagged PPase (custom-made) at $4{ }^{\circ} \mathrm{C}$ overnight. Ni-NTA 424 Superflow resin (Qiagen) was used to remove the cleaved His-tag and PPase. The final 425 sample was concentrated to about $5 \mathrm{mg} \mathrm{ml}^{-1}$. 
426 For the inactive mGlu7 homodimer, the STREP resin was washed with 10 column volumes

427 of wash buffer containing $25 \mathrm{mM}$ HEPES, $\mathrm{pH} 7.5,0.05 \%$ (w/v) DDM, 0.01\% (w/v) CHS,

$428150 \mathrm{mM} \mathrm{NaCl}$, and $50 \mu \mathrm{M}$ MMPIP. The detergent was exchanged to GDN following the

429 same protocol mentioned above. The protein was eluted by $200 \mathrm{mM}$ Tris, $\mathrm{pH} 8.0,0.01 \%(\mathrm{w} / \mathrm{v})$

$430 \mathrm{GDN}, 150 \mathrm{mM} \mathrm{NaCl}, 50 \mathrm{mM}$ biotin, and $50 \mu \mathrm{M}$ MMPIP, and concentrated to about $5 \mathrm{mg} \mathrm{ml}^{-}$ 4311.

432 The agonist/PAM-bound mGlu2 homodimer was purified following the same protocol of 433 inactive mGlu7 except that the ligands $50 \mu \mathrm{M}$ LY354740 and $50 \mu \mathrm{M}$ JNJ-40411813 were 434 used throughout the whole process.

435 Tandem affinity-chromatography was performed to prepare the mGlu2-7 heterodimer 436 sample. In brief, the TALON resin was washed with 10 column volumes of $25 \mathrm{mM}$ HEPES, $437 \mathrm{pH} 7.5,0.05 \%(\mathrm{w} / \mathrm{v}) \mathrm{DDM}, 0.01 \%(\mathrm{w} / \mathrm{v}) \mathrm{CHS}, 150 \mathrm{mM} \mathrm{NaCl}, 30 \mathrm{mM}$ imidazole, $50 \mu \mathrm{M}$ 438 NAM563, and $25 \mu \mathrm{M}$ MMPIP. Then, the detergent was exchanged to GDN and the protein 439 was eluted by $25 \mathrm{mM}$ HEPES, pH 7.5, 0.01\% (w/v) GDN, $150 \mathrm{mM} \mathrm{NaCl}, 300 \mathrm{mM}$ 440 imidazole, $50 \mu \mathrm{M}$ NAM563, and $25 \mu \mathrm{M}$ MMPIP. The elution was then incubated with the 441 STREP resin to remove mGlu2 homodimer. The final concentration of mGlu2-7 heterodimer 442 was about $7 \mathrm{mg} \mathrm{ml}^{-1}$.

\section{Expression and purification of DN13}

444 The nanobody DN13 was prepared as previously described ${ }^{9}$. In brief, DN13 was cloned into 445 the pET32a vector and transformed into E. coli BL21DE3 strain for expression. The

446 transformed cells were cultured in LB media supplemented with $100 \mu \mathrm{g} \mathrm{ml}^{-1}$ ampicillin, $4470.1 \%(\mathrm{w} / \mathrm{v})$ glucose and $1 \mathrm{mM} \mathrm{MgCl} 2$ at $37{ }^{\circ} \mathrm{C}$ until the $\mathrm{OD}_{600}$ reached 0.7 . Then the cells 448 were induced with $1 \mathrm{mM}$ IPTG and grown at $28^{\circ} \mathrm{C}$ overnight. The cells were collected by 
449 centrifugation at 4,000g for 15 min and resuspended in ice-cold TES buffer containing 200 $450 \mathrm{mM}$ Tris, $\mathrm{pH} 8.0,0.5 \mathrm{mM}$ EDTA, and $0.5 \mathrm{M}$ sucrose. After incubation at $4{ }^{\circ} \mathrm{C}$ for $2 \mathrm{~h}$, same 451 volume of a diluted TES buffer (TES buffer : double distilled water $=1: 3$ ) was added for

452 further incubation for $2 \mathrm{~h}$. The periplasmic extract was collected by centrifugation at $50,000 \mathrm{~g}$

453 for $30 \mathrm{~min}$ and incubated with the TALON resin at $4{ }^{\circ} \mathrm{C}$ overnight. The resin was then 454 washed with $20 \mathrm{mM}$ Tris, $\mathrm{pH} 8.0,100 \mathrm{mM} \mathrm{NaCl}$, and $30 \mathrm{mM}$ imidazole and eluted by the 455 same buffer supplemented with $300 \mathrm{mM}$ imidazole. The protein was concentrated to about 10 $456 \mathrm{mg} \mathrm{ml}^{-1}$ and stored at $-80{ }^{\circ} \mathrm{C}$ with addition of $20 \%$ glycerol until further use.

\section{Cryo-EM sample preparation, data acquisition and processing}

458 Using Vitrobot Mark IV (ThermoFisher), $3 \mu$ of protein sample were applied to glow459 discharged 300 mesh amorphous NiTi foil 1.2/1.3 (Au) ${ }^{26}$ (mGlu2 homodimers), Quantifoil 460 R1.2/1.3 (Au) (mGlu7 homodimer), or CryoMatrix R0.6/1 (Au) (mGlu2-7 heterodimer) grids.

461 The grids were blotted at $4{ }^{\circ} \mathrm{C}$ and $100 \%$ humidity for $4 \mathrm{~s}, 6 \mathrm{~s}$ and $1 \mathrm{~s}$, respectively, and 462 rapidly plunged into liquid ethane for vitrification.

For mGlu2, all datasets were collected on a 300-kV Titan Krios G2 electron microscope 464 (FEI) equipped with K2 Bioquantum direct electron detector (Gatan) and GIF Quantum 465 energy filter. The defocus range varied from $-1.2 \mu \mathrm{m}$ to $-2.2 \mu \mathrm{m}$ and the dose was 1.75 466 electrons per frame. 10,137 and 2,425 movies of inactive mGlu2 were collected by

467 SerialEM $^{27}$ with a pixel size of $0.82 \AA$ and $1.04 \AA$, respectively. A total of 6,989 movies of 468 the agonist/PAM-bound mGlu2 were collected with a pixel size of $1.04 \AA$. All movies were 469 subjected to beam-induced motion correction and dose-weighting using RELION's 470 implementation $^{28}$. Contrast transfer function (CTF) parameters for each micrograph were 471 estimated by Gctf $^{29}$. Gautomatch (developed by K. Zhang, MRC Laboratory of Molecular 472 Biology, Cambridge, UK; http:/www.mrc-lmb.cam.ac.uk/kzhang/Gautomatch/) was used to 
473 pick particles for topaz training ${ }^{30}$ and CryoSPARC ${ }^{31}$ was used to generate initial models.

474 Total 1,711,322 particles and 1,076,522 particles were picked for inactive and agonist/PAM-

475 bound mGlu2, respectively. 801,134 particles of inactive mGlu2 were subjected to three-

476 dimensional (3D) auto-refinement after 3D classification, followed by Bayesian polishing

477 and magnification CTF refinement to yield a 3.4- $\AA$ resolution map. Four rounds of TMD-

478 masked 3D classification were performed to improve the map quality of the TMDs and the

479 best class was selected to run 3D auto-refinement with C2 symmetry and Sidesplitter

480 refinement ${ }^{32}$. A 3.6- $\AA$ map was obtained. For the agonist/PAM-bound mGlu2, total 440,306

481 particles were selected after 3D classification. A similar data processing procedure was

482 followed as above. Final 365,101 particles were selected and subjected to several refinements

483 and yielded a 3.1- $\AA$ resolution map. Both maps were post-processed with LocSpiral ${ }^{33}$ and

484 further locally filtered according to the local resolution values for the following model

485 building and refinement.

486 The datasets of mGlu7 were collected on a $200-\mathrm{kV}$ Talos Arctica electron microscope 487 (FEI) equipped with K2 Bioquantum direct electron detector (Gatan) and GIF Quantum 488 energy filter. The defocus range varied from $-0.5 \mu \mathrm{m}$ to $-2.0 \mu \mathrm{m}$ and the dose was 1.47 489 electrons per frame. A total of 13,282 movies were collected by SerialEM ${ }^{27}$ with a physical 490 pixel size of $0.8 \AA$. All movies were subjected to beam-induced motion correction and dose491 weighting using RELION's implementation ${ }^{28}$. CTF parameters for each micrograph were 492 estimated by $\mathrm{Gctf}^{29}$. Particle selection was performed using template-free auto-picking in 493 RELION-3.0. A total of 3,051,969 particles were extracted and then subjected to three rounds 494 of reference-free two-dimensional classification and four rounds of 3D classification. The 495 best-looking classes with 1,011,214 particles were selected for 3D auto-refinement and 496 Bayesian polishing with C2 symmetry imposed to yield a 4.0- $\AA$ resolution map. Focused 497 refinement of the ECDs was performed, which further improved the ECD map resolution to 
$498 \quad 3.6$ A. A composite map was generated in UCSF Chimera ${ }^{34}$ using 'vop maximum' command 499 and used for model building and refinement.

500 For the mGlu2-7 heterodimer, the data were collected on a 300-kV Titan Krios G3 electron 501 microscope (FEI) equipped with K3 Summit direct electron detector (Gatan) and GIF 502 Quantum energy filter. The final magnified pixel size was $1.045 \AA$ in the super-resolution 503 counting mode. Each movie stack was dose-fractionated over 32 frames with the dose of 2.2 504 electrons per frame. The defocus values of all movie stacks varied from $-1.3 \mu \mathrm{m}$ to $-2.3 \mu \mathrm{m}$.

505 A total of 18,547 movies were collected by SerialEM ${ }^{27}$. Beam-induced motion and drift 506 correction were performed using MotionCor $2^{35}$. The CTF parameters for each micrograph 507 were determined by $\operatorname{Gctf}^{29}$. Particle selection was performed using template-free auto-picking 508 in RELION 3.0 (ref. 28). A total of 6,587,999 particles were extracted and then subjected to 509 three rounds of reference-free 2D classification and six rounds of 3D classification. The best 510 classes with $1,113,538$ particles were selected and subjected to $3 \mathrm{D}$ auto-refinement and 511 Bayesian polishing to get a $3.9-\AA$ resolution map. Focused refinement of the ECDs was 512 performed, which further improved the ECD map resolution to $3.5 \AA$. A composite map was 513 generated in UCSF Chimera ${ }^{34}$ using 'vop maximum' command and used for subsequent 514 model building and analysis. The maps of mGlu2 and mGlu7 homo- and heterodimers exhibit 515 a higher resolution in the VFT region compared to that in the CRDs and TMDs, indicating 516 that these regions are more structurally dynamic.

\section{Cryo-EM model building and refinement}

518 The structures of inactive mGlu2 and mGlu7 were built using the inactive mGlu5 structure 519 (PDB ID: 6N52) as an initial model. The structure of agonist/PAM-bound mGlu2 was built 520 using the agonist/PAM-bound mGlu5 structure (PDB ID: 6N51) as an initial model. The 521 model of the inactive mGlu2-7 heterodimer was built using the solved structures of inactive 
522 mGlu2 and mGlu7. All the models were docked into the cryo-EM density maps using

523 Chimera ${ }^{34}$, followed by iterative manual adjustment in $\operatorname{Coot}^{36}$ and real space refinement in

524 Phenix $^{37}$. The crystal structures of mGlu2-TMD-NAM complexes (see below for materials 525 and methods) were used to aid refinement of the TMDs in the inactive mGlu2 structure due 526 to a relatively low resolution of the cryo-EM map in this region. The model quality was 527 validated using MolProbity ${ }^{38}$. The final refinement statistics are provided in Extended Data

528 Table 1. The final models include most of residues in the receptors and the agonist LY354740 529 and nanobody DN13 in the agonist/PAM-bound mGlu2 structure. The mGlu2 NAM 530 NAM563 (inactive mGlu2 and mGlu2-7), mGlu7 NAM MMPIP (inactive mGlu7 and 531 mGlu2-7), mGlu2 PAM JNJ-40411813 (agonist/PAM-bound mGlu2), and the FKBP and 532 FRB fusion proteins in the $\mathrm{C}$ termini of the mGlu2-7 heterodimer were not modeled due to 533 the relatively low resolution in the TMD region.

\section{Expression, purification, and crystallization of mGlu2-TMD-NAM complexes}

535 The gene of mGlu2-TMD (residues 556-845) was modified by introducing the mutations $536 \mathrm{~N} 655^{3.52} \mathrm{Y}$ and $\mathrm{H} 815^{7.53} \mathrm{Y}$ and replacing residues $663-670$ at ICL2 with a fusion protein

537 flavodoxin $^{39}$, and cloned into a modified pFastBac1 vector with the HA signal sequence and 538 Flag-tag at the $\mathrm{N}$ terminus and the PPase site followed by a $10 \times$ His-tag at the $\mathrm{C}$ terminus. 539 The optimized mGlu2-TMD construct was expressed in Spodoptera frugiperda (Sf9) 540 (Invitrogen) insect cells using the Bac-to-Bac Baculovirus Expression System (Invitrogen). $541 S f 9$ cells at a cell density of $2 \times 10^{6}$ cells per ml were infected with high-titer virus at a MOI 542 (multiplicity of infection) of 5 . Cells were harvest $48 \mathrm{~h}$ post infection by centrifugation.

543 The cells expressing mGlu2-TMD were lysed in the hypotonic buffer containing $10 \mathrm{mM}$ 544 HEPES, pH 7.5, $10 \mathrm{mM} \mathrm{MgCl} 2,20 \mathrm{mM} \mathrm{KCl}$, and EDTA-free protease inhibitor cocktail 545 tablets (Roche). Extensive washing of the membranes was performed by repeated dounce 
546 homogenization and centrifugation in the hypotonic and high-osmotic buffers. Purified

547 membranes were suspended in the hypotonic buffer with addition of $30 \%$ glycerol, $20 \mu \mathrm{M}$

548 NAM563 (ref. 5) or NAM597 (ref. 10) (provided by Merck), and EDTA-free protease

549 inhibitor cocktail. After incubation at $4{ }^{\circ} \mathrm{C}$ for $1 \mathrm{~h}$, the complex was extracted by adding equal

550 volume of solubilization buffer containing $100 \mathrm{mM}$ HEPES, $\mathrm{pH} 7.5,1 \mathrm{M} \mathrm{NaCl}, 1 \%(\mathrm{w} / \mathrm{v})$

551 DDM, and $0.2 \%(\mathrm{w} / \mathrm{v}) \mathrm{CHS}$. After incubation at $4{ }^{\circ} \mathrm{C}$ for $3 \mathrm{~h}$, the supernatant was isolated by

552 centrifugation at $160,000 \mathrm{~g}$ for $30 \mathrm{~min}$ and incubated with TALON resin (Clontech) at $4{ }^{\circ} \mathrm{C}$

553 overnight. The resin was washed with 25 column volumes of wash buffer containing $25 \mathrm{mM}$

554 HEPES, pH 7.5, $500 \mathrm{mM} \mathrm{NaCl}, 0.05 \%$ (w/v) DDM, 0.01\% (w/v) CHS, 10\% glycerol, $50 \mu \mathrm{M}$

555 NAM563 (or NAM597), and $30 \mathrm{mM}$ imidazole, and eluted by increasing the concentration of

556 imidazole to $300 \mathrm{mM}$. PD MiniTrap G-25 column (GE Healthcare) was then used to remove

557 imidazole. The sample was supplemented with $100 \mu \mathrm{M}$ NAM563 (or NAM597) and

558 incubated with His-tagged PPase (custom-made) at $4{ }^{\circ} \mathrm{C}$ overnight to cut off His-tag. The Ni-

559 NTA resin (Qiagen) was added to remove the cleaved His-tag and PPase. The purified

560 sample was concentrated to $20-30 \mathrm{mg} \mathrm{ml}^{-1}$ with a $100-\mathrm{kDa}$ molecular weight cut-off

561 concentrator (Millipore).

562 The mGlu2-TMD-NAM complexes were crystallized using the lipidic cubic phase (LCP)

563 method $^{40}$. The proteins were mixed with lipid (monoolein/cholesterol 10:1 by mass) at a ratio

564 of 1:1.5 (w/w) using a syringe lipid mixer. The LCP mixture was dispensed onto 96-well

565 glass sandwich plates (Shanghai FAstal BioTech) in $35 \mathrm{nl}$ drop and overlaid with $800 \mathrm{nl}$ 566 precipitant solution using a Gryphon robot (Art-Robbins). The crystals of mGlu2-TMD-

567 NAM563 appeared in $100 \mathrm{mM}$ HEPES, pH 7.0, 100-200 mM NaCl, 100-200 mM MgCl 2 , 568 and $20-30 \%(\mathrm{v} / \mathrm{v})$ PEG 400 after 1 day and reached full size in 4-8 days. The crystals of 569 mGlu2-TMD-NAM597 grew in $100 \mathrm{mM}$ MES, pH 6.0-6.5, 50-200 mM NaCl, 50-150 mM $570 \mathrm{MgCl}_{2}, 20 \%-35 \%$ (v/v) PEG 400 and reached full size in 7 days. Crystals were harvested 
using 50-75 $\mu \mathrm{m}$ micro mounts (M2-L19-50/150, MiTeGen) and immediately flash-frozen in

572 liquid nitrogen.

573 X-ray data collection, processing, and structure determination of mGlu2-TMD-NAMs

574 Crystal diffraction data collection was performed at the SPring-8 beam line 41XU, Hyogo, 575 Japan, using a Pilatus3 6M detector (X-ray wavelength $1.0000 \AA$ ). The crystals were exposed 576 with a $10 \mu \mathrm{m} \times 8 \mu \mathrm{m}$ mini-beam for $0.2 \mathrm{~s}$ and $0.2^{\circ}$ oscillation per frame. Due to radiation 577 damage, data collection was limited to $10-15^{\circ}$ per crystal. HKL $2000^{41}$ was used for 578 integrating and scaling data from 21 mGlu2-TMD-NAM563 crystals and 26 mGlu2-TMD579 NAM597 crystals. Both structures were solved by molecular replacement with Phaser $^{42}$ using 580 mGlu1-TMD (PDB: 4OR2) and flavodoxin (PDB: 1I1O) structures as searching models. The 581 correct molecular replacement solutions contained one mGlu2-flavodoxin molecule in the 582 asymmetric unit. Refinements were performed with Refmac5 ${ }^{43}$, Buster ${ }^{44}$, and Phenix ${ }^{37}$ 583 followed by manual examination into $\left|2 F_{\mathrm{o}}\right|-\left|F_{\mathrm{c}}\right|$ and $\left|F_{\mathrm{o}}\right|-\left|F_{\mathrm{c}}\right|$ maps with Coot $^{36}$. The final 584 models of the mGlu2-TMD-NAM563 and mGlu2-TMD-NAM597 complexes contain 262 585 residues of mGlu2 (G564-V825) and 147 residues of flavodoxin (A1-I147). In both crystal 586 structures, the receptor TMDs form dimers through a dimer interface mediated by helices I,

587 VI, and VII, which is likely resulted from crystal packing.

\section{IP accumulation assay}

589 Flag-tagged wild-type and mutant mGlu2 and mGlu7 receptors were cloned into the pTT5 vector and expressed in HEK293F cells. Cells were co-transfected with the plasmids of

591 receptor, glutamate transporter $\mathrm{EAAC1}$, and a chimera $\mathrm{G}$ protein $\mathrm{G} \alpha_{\mathrm{q} i 9}$, which allows

592 efficient signalling through the phospholipase $\mathrm{C}$ pathway ${ }^{13}$, at a ratio of 2:1:2. Cells were

593 harvested $48 \mathrm{~h}$ post transfection. The surface expression of receptors was measured with the 594 Monoclonal Anti-Flag M2-FITC antibody (Sigma; 1:100 diluted in TBS+4\% BSA) using a 
595 flow cytometry reader (Millipore). IP1 accumulation was measured using IP-One $\mathrm{G}_{\mathrm{q}}$ assay kit 596 (Cisbio Bioassays) following the manufacturer's instructions.

597 For LY354740-induced IP accumulation of mGlu2, the cells expressing the wild-type 598 mGlu2 or mutants $(18,000$ cells per well) were incubated with increasing concentrations of 599 LY354740 $(1.58 \mathrm{pM}-25 \mu \mathrm{M})$ at $37^{\circ} \mathrm{C}$ for $45 \mathrm{~min}$. For glutamate-induced IP accumulation of $600 \mathrm{mGlu} 7$, the cells expressing the wild-type mGlu7 or mutants $(12,000$ cells per well) were 601 incubated with $2.5 \mu \mathrm{M}$ VU0422288 (mGlu7 PAM) ${ }^{45}$ and increasing concentrations of L602 glutamate $(1.58 \mu \mathrm{M}-100 \mathrm{mM})$ at $37^{\circ} \mathrm{C}$ for $1 \mathrm{~h}$. To measure the basal activity of mGlu2 and $603 \mathrm{mGlu}$, cells were incubated with stimulation buffer at $37{ }^{\circ} \mathrm{C}$ for $90 \mathrm{~min}$. Inhibition of IP 604 accumulation by the NAMs of mGlu2 and mGlu7 was measured by incubating the cells with 605 the agonist at $\mathrm{EC}_{80}$ concentration (100 $\mathrm{nM} \mathrm{LY354740} \mathrm{for} \mathrm{mGlu2;} 10 \mathrm{mM}$ glutamate for 606 mGlu7) and increasing concentrations of NAMs $(0.13 \mathrm{nM}-50 \mu \mathrm{M}$ NAM563; $0.76 \mathrm{nM}-50$

$607 \mu \mathrm{M}$ MMPIP) for $90 \mathrm{~min}$. Cells were then incubated with cryptate-labelled anti-IP1 608 monoclonal antibody (1:20 diluted in lysis and detection buffer) and IP1-d2 antibody (1:20 609 diluted in lysis and detection buffer) for $1 \mathrm{~h}$ at room temperature. Fluorescence signal was 610 measured using a Synergy II (Bio-Tek) plate reader with excitation at $330 \mathrm{~nm}$ and emission at

$611620 \mathrm{~nm}$ and $665 \mathrm{~nm}$. The accumulation of IP1 was calculated according to a standard dose-

612 response curve using GraphPad Prism 8.0 (GraphPad software). The basal activity was 613 calculated by subtracting the IP production measured in the control $\left(\mathrm{G}_{\mathrm{qi}}\right.$ alone $)$ for the WT 614 receptor and all the mutants.

\section{BRET assay using TRUPATH biosensors}

616 To measure $\mathrm{G}_{\mathrm{i}}$ protein activation, a BRET assay using TRUPATH biosensors ${ }^{24}$, which 617 measures the proximal interaction between the $\alpha$ and $\gamma$ subunits, was performed. In brief, 618 HEK293T cells (Invitrogen) were plated in 6-well plates at a density of $6-7 \times 10^{5}$ cells per 
619 well one day before transfection. Then, the cells were co-transfected with plasmids of 620 receptor, G $\alpha$-RLuc8, G $\beta$, and G $\gamma$-GFP2 [TRUPATH was a gift from Bryan Roth (Addgene

621 kit \# 1000000163)] at a ratio of 1:1:1:1 (500 ng per plasmid). After $24 \mathrm{~h}$ post transfection, 622 cells were digested by $0.25 \%$ Trypsin-EDTA (Thermo Fisher Scientific) and plated in poly623 D-lysine-coated white 96-well assay plates (Corning) at a density of $2-5 \times 10^{4}$ cells per well. 624 After $24 \mathrm{~h}$, the culture medium was replaced with $60 \mu \mathrm{l}$ of assay buffer $[1 \times$ Hank's balanced 625 salt solution (HBSS), $20 \mathrm{mM}$ HEPES, $\mathrm{pH}$ 7.4, and $0.1 \%$ BSA), followed by adding $10 \mu \mathrm{l}$ 626 freshly prepared $50 \mu \mathrm{M}$ coelenterazine 400a (Nanolight Technologies) and equilibrating for 5 627 min. Then, plates were measured by Synergy II (Bio-Tek) plate reader with $410 \mathrm{~nm}$ (RLuc8628 coelenterazine 400a) and $515 \mathrm{~nm}$ (GFP2) emission filters for $5 \mathrm{~min}$. The cells were then 629 treated with $30 \mu \mathrm{l}$ ligand for $5 \mathrm{~min}$ and continued to read serially 8 times. The last 630 measurements were used in all analyses. The BRET ratios were calculated as the ratio of the 631 GFP2 emission to RLuc8 emission.

\section{Intracellular calcium release measurement}

633 The Intracellular calcium release was measured as describe before ${ }^{21}$. In brief, the HEK293 634 cells (ATCC, CRL-1573) were transfected with the chimeric G protein $\mathrm{G}_{\mathrm{qi} 9}$ and the 635 glutamate transporter EAAC1 together with the mGlu constructs. The transfected cells were 636 loaded in 96-well plates with $1 \mu \mathrm{M}$ Fluo4-AM in fresh buffer (20 mM HEPES, pH 7.4, 130 $637 \mathrm{mM} \mathrm{NaCl}, 5.1 \mathrm{mM} \mathrm{KCl}, 0.42 \mathrm{mM} \mathrm{KH}_{2} \mathrm{PO}_{4}, 0.32 \mathrm{mM} \mathrm{Na}_{2} \mathrm{HPO}_{4}, 5.27 \mathrm{mM}$ glucose, $3.3 \mathrm{mM}$ $638 \mathrm{Na}_{2} \mathrm{CO}_{3}, 0.1 \% \mathrm{BSA}$, and $2.5 \mathrm{mM}$ probenecid) for $1 \mathrm{~h}$ at $37^{\circ} \mathrm{C}$. The fluorescence (excitation $639485 \mathrm{~nm}$, emission $525 \mathrm{~nm}$ ) was measured for $60 \mathrm{~s}$ by a FlexStation 3 microplate reader 640 (Molecular Devices, Sunnyvale, CA, USA). The ligand was injected at $20 \mathrm{~s}$ during recording. 
642 HEK293 cells were transiently transfected with indicated plasmids and plated in 96-well 643 plates at $10^{5}$ cells per well. After $24 \mathrm{~h}$ transfection, the cells were washed with PBS and 644 incubated with fluorophore-conjugated antibodies HA-Tb cryptate antibody (Pekin Elmer; 645 1:160 diluted in Tris KREBS buffer) and Flag-d2 antibody (Pekin Elmer; 1:267 diluted in 646 Tris KREBS buffer) overnight at 4 . The fluorescence of the cryptate $(620 \mathrm{~nm})$ and $\mathrm{d} 2(665$ $647 \mathrm{~nm}$ ) was measured $40 \mu \mathrm{s}$ after excitation at $337 \mathrm{~nm}$ using PheraStar instrument (BMG 648 Labtechnologies $)$. tr-FRET signals were expressed as $\Delta \mathrm{F} \%=\left[(665 / 620)_{\text {sample }}-(665 / 620)_{\text {mock }}\right]$ $649 \times 100 /(665 / 620)_{\text {mock}}$.

650 The expression levels of different proteins were evaluated by ELISA. The transfected cells 651 were fixed for 5 min with paraformaldehyde (4\%), then blocked with PBS containing $1 \%$ 652 fetal bovine serum (FBS) and incubated for $30 \mathrm{~min}$ with monoclonal anti-HA horseradish 653 peroxidase (Roche; 1:2,000 diluted in PBS $+1 \%$ FBS) or anti-Flag horseradish peroxidase 654 (Sigma; 1:20,000 diluted in PBS+1\% FBS). Bound antibody was detected using a 655 SuperSignal substrate (Pierce) and a Tecan infinite 200Pro plate reader.

\section{Cysteine crosslinking and fluorescent-labelled blot experiments}

657 Crosslinking of mGlu2 and mGlu2-7 mutants were measured by fluorescent-labelled blot as 658 previously described ${ }^{13}$. After $48 \mathrm{~h}$ transfection, the HEK293 cells plated in 12-well plates 659 were incubated in GlutaMAX medium (Gibco) overnight. Then, the cells were labelled with 660100 nM SNAP-Surface 647 (NEW ENGLAND BioLabs Inc.) in culture medium at $37{ }^{\circ} \mathrm{C}$ for

$6611 \mathrm{~h}$. Cells were then washed once with PBS and crosslinked with $1.5 \mathrm{mM}$ CuP in crosslinking 662 buffer $\left(16.7 \mathrm{mM}\right.$ Tris, $\mathrm{pH} 8.0,100 \mathrm{mM} \mathrm{NaCl}, 1 \mathrm{mM} \mathrm{CaCl}_{2}$, and $5 \mathrm{mM} \mathrm{MgCl}_{2}$ ) at room 663 temperature for $20 \mathrm{~min}$. After incubation with $10 \mathrm{mM} \mathrm{N}$-ethylmaleimide at $4{ }^{\circ} \mathrm{C}$ for $15 \mathrm{~min}$ to 664 stop the crosslinking reaction, cells were lysed with lysis buffer (50 mM Tris, $\mathrm{pH} 7.4,150$ $665 \mathrm{mM} \mathrm{NaCl}, 1 \%$ Nonidet P-40, and $0.5 \%$ sodium deoxycholate) at $4{ }^{\circ} \mathrm{C}$ for $1 \mathrm{~h}$. After 
centrifugation at $12,000 \mathrm{~g}$ for $30 \mathrm{~min}$ at $4{ }^{\circ} \mathrm{C}$, supernatants were mixed with loading buffer at

$66737^{\circ} \mathrm{C}$ for $10 \mathrm{~min}$. Equal amounts of proteins were resolved by $6 \%$ SDS-PAGE. Proteins were

668 transferred to nitrocellulose membranes (Millipore). Membranes were imaged by Odyssey

669 CLx imager (LI-COR Bioscience, Lincoln, NE, USA).

\section{Data availability}

671 Atomic coordinates and cryo-EM density maps for the structures of inactive mGlu2, 672 agonist/PAM-bound mGlu2, inactive mGlu7, and inactive mGlu2-7 have been deposited in 673 the RCSB Protein Data Bank (PDB) under accession codes 7EPA, 7EPB, 7EPC, and 7EPD, 674 and the Electron Microscopy Data Bank (EMDB) under accession codes EMD-31235, EMD675 31236, EMD-31237, and EMD-31238. Atomic coordinates for the structures of mGlu2676 TMD-NAM563 and mGlu2-TMD-NAM597 have been deposited in the RCSB PDB under 677 accession codes 7EPE and 7EPF. The uncropped gels shown in Extended Data Fig. 1r, u, aa 678 are displayed in Supplementary Figure 1.

\section{References}

68024 Olsen, R. H. J. et al. TRUPATH, an open-source biosensor platform for interrogating the 681 GPCR transducerome. Nat. Chem. Biol. 16, 841-849 (2020).

68225 Fegan, A., White, B., Carlson, J. C. \& Wagner, C. R. Chemically controlled protein assembly: 683 techniques and applications. Chem. Rev. 110, 3315-3336 (2010).

68426 Huang, X. et al. Amorphous nickel titanium alloy film: A new choice for cryo electron

69029 Zhang, K. Gctf: Real-time CTF determination and correction. J. Struct. Biol. 193, 1-12 (2016).

$69130 \quad$ Bepler, T. et al. Positive-unlabeled convolutional neural networks for particle picking in cryo692 electron micrographs. Nat. Methods 16, 1153-1160 (2019).

$69331 \quad$ Punjani, A., Rubinstein, J. L., Fleet, D. J. \& Brubaker, M. A. cryoSPARC: algorithms for 694 rapid unsupervised cryo-EM structure determination. Nat. Methods 14, 290-296 (2017).

69532 Ramlaul, K., Palmer, C. M., Nakane, T. \& Aylett, C. H. S. Mitigating local over-fitting during 696 single particle reconstruction with SIDESPLITTER. J. Struct. Biol. 211, 107545 (2020).

33 Kaur, S. et al. Local computational methods to improve the interpretability and analysis of cryo-EM maps. Nat. Commun. 12, 1240 (2021).

34 Pettersen, E. F. et al. UCSF Chimera--A Visualization System for Exploratory Research and Analysis. J. Comput. Chem. 25, 1605-1612 (2004). 
70135 Zheng, S. Q. et al. MotionCor2: anisotropic correction of beam-induced motion for improved

36 Emsley, P., Lohkamp, B., Scott, W. G. \& Cowtan, K. Features and development of Coot. Acta Crystallogr. D Biol. Crystallogr. 66, 486-501 (2010).

37 Adams, P. D. et al. PHENIX: a comprehensive Python-based system for macromolecular structure solution. Acta Crystallogr. D Biol. Crystallogr. 66, 213-221 (2010).

38 Chen, V. B. et al. MolProbity: all-atom structure validation for macromolecular crystallography. Acta Crystallogr. D Biol. Crystallogr. 66, 12-21 (2010).

39 Chun, E. et al. Fusion Partner Toolchest for the Stabilization and Crystallization of G ProteinCoupled Receptors. Structure 20, 967-976 (2012).

40 Caffrey, M. \& Cherezov, V. Crystallizing Membrane Proteins Using Lipidic Mesophases. Nat. Protoc. 4, 706-731 (2009).

41 Otwinowski, Z. \& Minor, W. Processing of X-ray diffraction data collected in oscillation mode. Methods Enzymol. 276, 307-326 (1997).

42 McCoy, A. J. et al. Phaser crystallographic software. J. Appl. Crystallogr. 40, 658-674 (2007).

43 Vagin, A. A. et al. REFMAC5 dictionary: organization of prior chemical knowledge and guidelines for its use. Acta Crystallogr. D Biol. Crystallogr. 60, 2184-2195 (2004).

44 Smart, O. S. et al. Exploiting structure similarity in refinement: automated NCS and targetstructure restraints in BUSTER. Acta Crystallogr. D Biol. Crystallogr. 68, 368-380 (2012).

45 Jalan-Sakrikar, N. et al. Identification of positive allosteric modulators VU0155094 (ML397) and VU0422288 (ML396) reveals new insights into the biology of metabotropic glutamate receptor 7. ACS Chem. Neurosci. 5, 1221-1237 (2014).

Acknowledgements The cryo-EM studies were performed at the Center for Biological and the EM facility of Shanghai Institute of Materia Medica (SIMM), Chinese Academy of

727 Sciences. We thank B. Zhu, X. Huang, X. Li, L. Chen, and X. Niu from CBI and Q. Wang

728 from SIMM for their help with cryo-EM data collection. The synchrotron radiation

729 experiments were performed at the BL41XU of SPring-8 with approval of the Japan

730 Synchrotron Radiation Research Institute (Proposal no. 2019A2543, 2019B2543, 2019A2541,

731 and 2019B2541). We thank the beamline staff members K. Hasegawa, N. Mizuno, T.

732 Kawamura, and H. Murakami of the BL41XU for help with X-ray data collection. We thank

733 Merck for providing the mGlu2 NAMs NAM563 and NAM597. This work was supported by

734 the National Key R\&D Program of China 2018YFA0507000 (Q.Z., B.W., and J.L.), National

735 Science Foundation of China grants 31825010 (B.W.), 81525024 (Q.Z.), 31830020 (F.S.),

736 and 81720108031 (J.L.), and CAS Strategic Priority Research Programs XDB37030100 (Q.Z.

737 and B.W.) and XDB37040102 (F.S.). 
738 Author contributions J.D. developed the protein expression and purification procedures of 739 mGlu2, prepared the mGlu2 protein samples for cryo-EM and crystallization, solved the 740 crystal structures of mGlu2-TMD-NAMs, performed IP accumulation assay, and helped with

741 manuscript preparation. D.W. developed the protein expression and purification procedures 742 of mGlu7, prepared the mGlu7 protein sample for cryo-EM, performed model building and 743 structure refinement of the homodimers, and helped with the IP accumulation assay and 744 manuscript preparation. H.F. performed the cryo-EM studies of the mGlu2 homodimers. C.X. 745 performed crosslinking, calcium release, and FRET assays. L.T. performed the cryo-EM 746 studies of the mGlu7 homodimer. S.L. developed the protein expression and purification 747 procedures of the mGlu2-7 heterodimer, prepared the mGlu2-7 protein sample for cryo-EM, 748 and performed model building and structure refinement of the heterodimer. S.H. performed 749 the cryo-EM studies of the mGlu2-7 heterodimer. Q.T. performed BRET assay. X.W. and 750 T.X. helped with protein preparation and functional assays. H.Z. collected the crystal 751 diffraction data. X.C. and C.Y. expressed the proteins. P.L. and Xi.W. helped with 752 crosslinking and calcium release assays. J.-P.P. and P.R. helped with data 753 analysis/interpretation and edited the manuscript. Y.Z. and H.L. provided mGlu2 ligands for 754 structural and functional studies. J.L. oversaw crosslinking, calcium release, and FRET 755 assays, helped with data analysis/interpretation, and edited the manuscript. F.S. oversaw the 756 cryo-EM studies of mGlu2 and mGlu7 homodimers, helped with data analysis/interpretation, 757 and edited the manuscript. B.W. and Q.Z. initiated the project, planned and analyzed 758 experiments, supervised the research, and wrote the manuscript with input from all co759 authors.

760 Competing interests The authors declare no competing interests.

761 Reprints and permissions information is available at http://www.nature.com/reprints. 
762 Correspondence and requests for materials should be addressed to H.L., J.L., F.S., B.W.

763 or Q.Z. 
and heterodimers. a, Comparison of the mGlu2 homodimers with different C-terminal truncations. The curves of size-exclusion chromatography (SEC) of purified protein samples show higher yield and better homogeneity for the protein with the C-terminal residues V826L872 truncated. b, Comparison of the mGlu7 homodimers with different C-terminal truncations. The SEC curves of purified protein samples show higher yield and better homogeneity for the protein with the C-terminal residues K860-I915 truncated. c, Comparison of the mGlu2 homodimers with different mutations. The SEC curves of purified protein samples show higher yield for the mutant $\mathrm{N} 655^{3.52} \mathrm{Y} / \mathrm{H} 815^{7.53} \mathrm{Y}$ (construct 1 , used to determine the inactive mGlu2 structure). d, Comparison of the mGlu7 homodimers with different mutations. The SEC results of purified protein samples show higher yield for the mutant $\mathrm{N} 678^{3.52} \mathrm{Y} / \mathrm{G} 722^{4.52} \mathrm{I} / \mathrm{I} 775^{5.59} \mathrm{~F} / \mathrm{P} 789^{6.38} \mathrm{Y}$ (construct 2, used to determine the inactive mGlu7 structure). e, LY354740-induced IP accumulation assay of wild-type (WT) mGlu2 and mutants. The IP accumulation data are shown as mean \pm SEM from at least three independent experiments performed in technical triplicate (same for below). The numbers of independent experiments (n) are shown in the parentheses (same for below). Surface expression levels of the mutants are reported as percent compared to the WT from at least three independent measurements performed in duplicate ( $\%$ of WT): N655 ${ }^{3.52} \mathrm{Y}, 112 \pm 29$; $\mathrm{H} 815^{7.53} \mathrm{Y}, 56 \pm 10$; S601A, $88 \pm 13$; C-truncation (V826-L872), $53 \pm 3$. f, Inhibition of LY354740-induced IP accumulation of WT mGlu2 and mutants by NAM563. g, Glutamateinduced IP accumulation assay of WT mGlu7 and mutants in presence of VU0422288.

786 Surface expression levels of the mutants (\% of WT): N678 ${ }^{3.52} \mathrm{Y}, 200 \pm 25 ; \mathrm{G} 722^{4.52} \mathrm{I}, 144 \pm$

$78714 ; \mathrm{I} 775^{5.59} \mathrm{~F}, 136 \pm 13 ; \mathrm{P} 789^{6.38} \mathrm{Y}, 129 \pm 12 ;$ C-truncation (K860-1915), $110 \pm 10 . \mathbf{h}$, 788 Inhibition of glutamate-induced IP accumulation of WT mGlu7 and mutants by MMPIP. i, 
789 LY354740-induced $\mathrm{G}_{\mathrm{i}}$ activation of WT mGlu2 and mutants measured by the BRET assay.

790 The BRET data are shown as mean \pm SEM from at least three independent experiments

791 performed in technical duplicate (same for below). j, Glutamate-induced $\mathrm{G}_{\mathrm{i}}$ activation of WT

792 mGlu7 and mutants in presence of VU0422288 measured by the BRET assay. k, Ligand

793 screening for the inactive mGlu2 homodimer. The SEC curves show that the NAM563-bound

794 mGlu2 has higher yield and better homogeneity compared to the apo receptor and

795 LY341495-bound mGlu2. 1, Ligand screening for the inactive mGlu7 homodimer. The SEC

796 curves show that the NAM MMPIP-bound mGlu7 has higher yield and better homogeneity

797 compared to the apo receptor and the receptor bound to other ligands. $\mathbf{m}$, Inhibition of

798 LY354740-induced IP accumulation of WT mGlu2 by NAM563 or NAM597. n, Comparison

799 of WT mGlu2 and construct 3 (used to determine the agonist/PAM-bound mGlu2 structure).

800 The SEC curves of purified protein samples show higher yield and better homogeneity for

801 construct 3. o, JNJ-40411813-induced IP accumulation assay of WT mGlu2 and mutants. p,

802 JNJ-40411813-induced $\mathrm{G}_{\mathrm{i}}$ activation of WT mGlu2 and mutants measured by the BRET

803 assay. q, Ligand screening for the agonist/PAM-bound mGlu2 homodimer. The SEC curves

804 show higher yield for the agonist- or PAM-bound receptor than the apo protein. $\mathbf{r}$, Nu-PAGE

805 and western-blot results of the mGlu2-7 heterodimer. Double staining (anti-His for his-tagged

806 mGlu2 and anti-Strep for Strep-tagged mGlu7) confirms the existence of mGlu2-7

807 heterodimer. Three independent experiments were performed with similar results. Results

808 from a representative experiment are shown. For gel source data, see Supplementary Figure 1.

$809 \mathbf{s}$, t , Glutamate-induced IP accumulation of WT mGlu2 and mutants. See Extended Data

810 Table 3 for detailed independent experiment numbers (n), statistical evaluation, and

811 expression level. u, Crosslinking of the mGlu2 mutants $\mathrm{C} 121 \mathrm{~A} / \mathrm{L} 698^{4.51} \mathrm{C}, \mathrm{C} 121 \mathrm{~A} / \mathrm{V} 699^{4.52} \mathrm{C}$,

812 and $\mathrm{C} 121 \mathrm{~A} / \mathrm{V} 782^{6.59} \mathrm{C}-\mathrm{C} 121 \mathrm{~A} / \mathrm{V} 789^{7.27} \mathrm{C}$ with $(+)$ or without (-) preincubation with the

813 agonist LY354740 and PAM JNJ-40411813 or the antagonist LY341495 and NAM Ro64- 
814 5299. The mutant $\mathrm{C} 121 \mathrm{~A} / \mathrm{V} 7822^{6.59} \mathrm{C}-\mathrm{C} 121 \mathrm{~A} / \mathrm{V} 789^{7.27} \mathrm{C}$ was tested using an optimized

$815 \mathrm{GABA}_{\mathrm{B}}$ quality control system, in which the C-terminal tails of the two mGlu2 subunits were

816 replaced by the modified $\mathrm{C} 1$ and $\mathrm{C} 2$ domains of $\mathrm{GABA}_{\mathrm{B} 1}$ and $\mathrm{GABA}_{\mathrm{B} 2}$, respectively, to only

817 allow the mGlu2 dimers containing the C1-C2 dimer reach the cell surface ${ }^{15}$. The WT and

818 mutant C121A were tested in parallel as controls. Three independent experiments were

819 performed with similar results. Results from a representative experiment are shown. For gel

820 source data, see Supplementary Figure 1. v, LY354740-induced IP accumulation assay of

821 mGlu2 in presence of the oxidant CuP. Surface expression levels of the mGlu2 mutants are

822 reported as percent compared to the WT from at least three independent measurements

823 performed in duplicate (\% of WT): C121A, $100 \pm 6 ; \mathrm{C} 121 \mathrm{~A} / \mathrm{L} 698^{4.51} \mathrm{C}, 84 \pm 11$;

$824 \mathrm{C} 121 \mathrm{~A} / \mathrm{V} 699^{4.52} \mathrm{C}, 92 \pm 9$. w, LY354740-induced IP accumulation assay of the mGlu2

825 mutants in the CRD. See Extended Data Table 3 for detailed statistical evaluation and

826 expression level. $\mathbf{x}$, Glutamate-induced IP accumulation assay of WT mGlu7 and mutants in

827 presence of VU0422288. See Extended Data Table 3 for detailed statistical evaluation and

828 expression level. $\mathbf{y}$, LY354740-induced IP accumulation assay of mGlu2 mutants in the CRD

829 and ECL2. See Extended Data Table 3 for detailed independent experiment numbers (n),

830 statistical evaluation, and expression level. z, JNJ-40411813-induced IP accumulation assay

831 of WT mGlu2 and the mutant in ECL2. aa, Crosslinking of the mGlu2-7 mutants

832 C121A/D174C (mGlu2)-C136A/R191C (mGlu7) and C121A/S176C (mGlu2)-

833 C136A/R191C (mGlu7). The crosslinking studies were carried out using the $\mathrm{GABA}_{\mathrm{B}} \mathrm{C} 1-\mathrm{C} 2$

834 quality control system that allows cell surface targeting of the heterodimer only ${ }^{21}$. The WT

835 heterodimer and mutant C121A (mGlu2)-C136A (mGlu7) were tested in parallel as controls.

836 Four independent experiments were performed with similar results. Results from a

837 representative experiment are shown. For gel source data, see Supplementary Figure 1. 
838 Extended Data Fig. 2 I Cryo-EM processing and 3D reconstruction workflow. a-f,

839 Results of the inactive mGlu2 homodimer. a, Processing workflow. b, Representative cryo-

840 EM image from four independent experiments with similar results. c, 2D averages. d, Cryo-

841 EM map colored according to local resolution $(\AA)$. e, Gold-standard FSC curve showing an

842 overall resolution at $3.6 \AA$ A. f, Cross-validation of model to cryo-EM density map. FSC curves

843 for the final model versus the final map and half maps are shown in black, red, and green,

844 respectively. g-l, Results of the inactive mGlu7 homodimer. g, Processing workflow. h,

845 Representative cryo-EM image from five independent experiments with similar results. i, 2D

846 averages. j, Cryo-EM map colored according to local resolution $(\AA)$. $\mathbf{k}$, Gold-standard FSC

847 curves showing an overall resolution at $4.0 \AA$ and a resolution at $3.6 \AA$ for the ECDs. $\mathbf{l}$,

848 Cross-validation of model to cryo-EM density map. m-r, Results of the agonist/PAM-bound

849 mGlu2 homodimer. m, Processing workflow. n, Representative cryo-EM image from three

850 independent experiments with similar results. o, 2D averages. p, Cryo-EM map colored

851 according to local resolution $(\AA)$. q, Gold-standard FSC curve showing an overall resolution

852 at $3.1 \AA$. $\mathbf{r}$, Cross-validation of model to cryo-EM density map. s-x, Results of the inactive

853 mGlu2-7 heterodimer. s, Processing workflow. t, Representative cryo-EM image from four

854 independent experiments with similar results. u, 2D averages. v, Cryo-EM map colored

855 according to local resolution $(\AA)$. w, Gold-standard FSC curves showing an overall resolution

856 at $3.9 \AA$ and a resolution at $3.5 \AA$ for the ECDs. $\mathbf{x}$, Cross-validation of model to cryo-EM

857 density map.

858 Extended Data Fig. 3 I Cryo-EM density maps of mGlu2 and mGlu7 homo- and

859 heterodimers. The models are shown as cartoon and sticks. The density maps are colored 860 gray. a, Map and model of the inactive mGlu2 structure shown for all transmembrane helices,

861 B and C helices in VFTs, CRDs, and intersubunit disulfide bond. The model is colored blue

862 (molecule A) and gray (molecule B). b, Map and model of the inactive mGlu7 structure 
863 shown for all transmembrane helices, B and C helices in VFTs, CRDs, and intersubunit

864 disulfide bond. The model is colored orange (molecule A) and yellow (molecule B). c, Map

865 and model of the agonist/PAM-bound mGlu2 structure shown for all transmembrane helices,

866 B and C helices in VFTs, LY354740, DN13, CRDs, and intersubunit disulfide bond. The

867 model is colored blue (molecule A) and gray (molecule B). d, Map and model of the inactive

868 mGlu2-7 structure shown for all transmembrane helices, B and C helices in VFTs, CRDs,

869 intersubunit disulfide bond, and some non-conserved residues in the two subunits that aided

870 modelling. The model is colored blue (mGlu2) and orange (mGlu7).

871 Extended Data Fig. 4 I VFT and CRD conformations in mGlus. a, Comparison of

872 intersubunit disulfide bonds in the inactive mGlu2 and mGlu7 homo- and heterodimers. The

873 structures of inactive mGlu2 homodimer, mGlu7 homodimer, and mGlu2-7 heterodimer are

874 shown in cartoon representation. The disulfide bonds are shown as yellow sticks. The

875 intersubunit disulfide bonds are highlighted by a red dashed box. b, Comparison of

876 intersubunit disulfide bonds in the inactive mGlu2 and agonist/PAM-bound mGlu2. The

877 structures of inactive mGlu2 homodimer and agonist/PAM-bound mGlu2 homodimer are

878 shown in cartoon representation. c, Comparison of VFT conformations in inactive mGlu

879 structures. The structures of inactive mGlu2, inactive mGlu5 (PDB ID: 6N52), inactive

880 mGlu7, apo mGlu2-VFT (PDB ID: 5KZN), and LY3020371 (antagonist)-bound mGlu2-VFT

881 (PDB ID: 5KZQ) are shown in cartoon representation. d, Comparison of the VFTs in the

882 inactive structures of mGlu2, mGlu5, and mGlu7. The structures are shown in a side view.

883 The $\mathrm{C} \alpha$ atoms of the mGlu2 residue $\mathrm{C} 234$ and its counterparts in mGlu5 and mGlu7 are

884 shown as spheres. The green arrow indicates the shift of the VFT in the mGlu5 structure

885 compared to that in the mGlu2 and mGlu7 structures. e, Comparison of the CRDs in the

886 inactive structures of mGlu2, mGlu5, and mGlu7. The structures are shown in both side and

887 extracellular views. The $\mathrm{C} \alpha$ atoms of the mGlu2 residue $\mathrm{C} 540$ and its counterparts in mGlu5 
and mGlu7 are shown as spheres. The distance between the $\mathrm{C} \alpha$ atoms of the cysteine residues

889 in each structure is indicated by a dashed line and colored blue (mGlu2), magenta (mGlu5),

890 or orange (mGlu7). The red arrow indicates the movement of the CRDs in mGlu5 and mGlu7

891 relative to that in mGlu2. f, Comparison of VFT conformations in agonist-bound mGlu

892 structures. The structures of LY354740/JNJ-40411813-bound mGlu2, L-quisqualate/CDPPB-

893 bound mGlu5 (PDB ID: 6N51), LY354740-bound mGlu2-VFT (PDB ID: 4XAQ), and

894 glutamate-bound mGlu2-VFT (PDB ID: 5CNI) are shown in cartoon representation and

895 colored blue, magenta, red, and light gold, respectively. g, Comparison of the CRDs in the

896 agonist/PAM-bound mGlu2 and mGlu5 structures. The structures are shown in both side and

897 extracellular views. h, Interactions between the CRDs in the agonist/PAM-bound mGlu2

898 structure. The residues that are involved in dimerization are shown as blue and gray sticks,

899 respectively. The disulfide bonds are shown as yellow sticks. i, Comparison of VFT

900 conformations in the inactive mGlu2 and mGlu7 homo- and heterodimers. $\mathbf{j}$, VFT dimer

901 interface in the inactive mGlu2-7 heterodimer structure. The residues that are involved in

902 dimerization are shown as sticks.

903 Extended Data Fig. 5 I Sequence alignment of the TMDs of human mGlus. Colors

904 represent the similarity of residues: red background, identical; red text, strongly similar. The

905 red arrows indicate the positions with mutations in the inactive mGlu2 and mGlu7 structures

906 (2, mGlu2; 7, mGlu7). The mGlu2 residues that are involved in TMD dimerization in the

907 inactive mGlu2 structure are indicated by green arrows. The conserved residues with the

908 modified Ballesteros-Weinstein numbers for class C GPCRs, 1.50, 2.50, 3.50, 4.50, 5.50,

9096.50 , and 7.50, are indicated by black arrows. The alignment was generated using UniProt

910 (http://www.uniprot.org/align/) and the graphic was prepared on the ESPript 3.0 server

911 (http://espript.ibcp.fr/ESPript/cgi-bin/ESPript.cgi). 
912 Extended Data Fig. 6 I Inter- and intrasubunit interactions upon mGlu2 activation and

913 NAM-binding sites in mGlus. a, Interactions between the CRD and ECL2 in the

914 agonist/PAM-bound mGlu2 and mGlu5 structures. The structures of agonist/PAM-bound

915 mGlu2 and mGlu5 (PDB ID: 6N51) are shown in cartoon representation and colored blue and

916 pink, respectively. The residues that potentially form interactions between the CRD and

917 ECL2 are shown as sticks. b, Comparison of the helical bundles in the agonist/PAM-bound

918 structures of mGlu2 and mGlu5. The mGlu2 residues $\mathrm{V} 782^{6.59}$ and $\mathrm{V} 789^{7.27}$ that are involved

919 in dimerization are shown as sticks. The red arrows indicate the movement of each helix in

920 the mGlu2 structure relative to the mGlu5 structure. c, Crystal structures of mGlu2-TMD-

921 NAMs. The receptor is colored cyan. The NAMs NAM563 and NAM597 are shown as sticks

922 and colored yellow and magenta, respectively. The mGlu2 residues that form interactions

923 with the NAMs are shown as cyan sticks (middle panels). The ligand electron densities are

924 contoured at $1.0 \sigma$ from an $\left|2 F_{\mathrm{o}}\right|-\left|F_{\mathrm{c}}\right|$ map, and colored gray (bottom panels). d, Comparison

925 of ligand-binding sites in mGlus and class A GPCRs. The ligands in the crystal structures of

926 mGlu2-TMD-NAM563, mGlu1-TMD-FITM (PDB ID: 4OR2), mGlu5-TMD-mavoglurant

927 (PDB ID: 4OO9), $\beta_{2} \mathrm{AR}-$ carazalol (PDB ID: 2RH1), M2-AF-DX384 (PDB ID: 5ZKB), and

928 D3R-eticlopride (PDB ID: 3PBL) are shown as sticks. Only the receptor in the mGlu2-

929 TMD-NAM563 structure is shown in cyan cartoon representation for clarity. e, Comparison

930 of the helical bundles in the inactive and active structures of mGlu2. The helical bundles in

931 the structures of mGlu2-TMD-NAM563, inactive mGlu2, agonist/PAM-bound mGlu2, and

932 the $\mathrm{G}_{\mathrm{i}}$-free (free) and $\mathrm{G}_{\mathrm{i}}$-bound subunits $(\mathrm{G})$ in the $\mathrm{mGlu}_{2}-\mathrm{G}_{\mathrm{i}}$ complex are shown in an

933 intracellular view. f, Comparison of helix VI in the mGlu2-TMD-NAM563 and mGlu2- $\mathrm{G}_{\mathrm{i}}$

934 structures. The red arrow indicates the downward shift of helix VI in the $\mathrm{G}_{\mathrm{i}}$-bound subunit of

935 the mGlu2 $-\mathrm{G}_{\mathrm{i}}$ complex relative to the mGlu2-TMD-NAM563 structure and the $\mathrm{G}_{\mathrm{i}}$-free

936 subunit in the $\mathrm{G}_{\mathrm{i}}$-bound structure. $\mathrm{g}$, Comparison of the $\mathrm{W}^{6.50}$ conformation in the inactive 
937 and active mGlu2 structures. The mGlu2-TMD-NAM563 structure and the $\mathrm{G}_{\mathrm{i}}$-bound subunit

938 in the mGlu2- $\mathrm{G}_{\mathrm{i}}$ structure are colored cyan and dark red, respectively. The residue W773 ${ }^{6.50}$

939 in the two structures and the residues that form interactions with W773 6.50 in the mGlu2-

940 TMD-NAM563 structure are shown as sticks. The red arrow indicates the rotamer

941 conformational change of $\mathrm{W}_{773^{6.50}}$ in the active structure relative to the inactive structure. $\mathbf{h}$,

942 Comparison of the ionic locks in the inactive and active mGlu2 structures. The mGlu2-

943 TMD-NAM563 structure is colored cyan. The $\mathrm{G}_{\mathrm{i}}$-bound subunit and $\mathrm{G}_{\mathrm{i}}$ protein in the 944 mGlu2 $-\mathrm{G}_{\mathrm{i}}$ structure are colored dark red and green, respectively. The residues $\mathrm{K} 653^{3.50}$,

$945 \mathrm{R} 656^{3.53}$, E754, and E758 $8^{6.35}$ that form ionic interactions in the inactive structure are shown

946 as sticks in both structures. The ionic interactions in the inactive structure are shown as red

947 dashed lines and the interaction between $\mathrm{R} 656^{3.53}$ and $\mathrm{G} \alpha_{\mathrm{i}}$ in the active structure is shown as

948 a blue dashed line.

949 Extended Data Fig. 7 I tr-FRET and functional assays of mGlu2 and mGlu7 homo- and

950 heterodimers. a, Validation of mGlu2-7 heterodimer and construct used for structural 951 determination by tr-FRET assay. The signals were measured using the N-terminal HA- and 952 Flag-tags in each subunit. In each panel, cartoons illustrating the dimer compositions are 953 shown at the bottom. Data are displayed as mean \pm SEM (bars) from three independent 954 experiments performed in technical triplicate with individual data points shown (dots). b, 955 Cell surface expression of homo- and heterodimers quantified by ELISA. Data were 956 calculated as the fold of mock. Data are displayed as mean \pm SEM (bars) from three 957 independent experiments performed in technical triplicate with individual data points shown 958 (dots). c-g, Calcium release assays of mGlu2-7 heterodimer. Data are shown as mean \pm SEM 959 from at least three independent experiments performed in technical triplicate and correspond 960 to the peak response. The numbers of independent experiments (n) are shown in the 961 parentheses. c, e, and f, LY354740-induced calcium release of wild-type (WT) mGlu2-7 
962 heterodimer and mutants. d, g, L-AP4-induced calcium release of WT mGlu2-7 heterodimer

963 and mutants. h, LY354740-induced $\mathrm{G}_{\mathrm{i}}$ activation of mGlu2-7 $7^{\times}$in presence of LY354740,

964 LY354740+mGlu2 PAM (JNJ-40411813), or LY354740+mGlu7 NAM (MMPIP,

965 ADX71743, or MDIP) measured by the BRET assay. Data are shown as mean \pm SEM from

966 at least three independent experiments performed in technical duplicate. The numbers of

967 independent experiments (n) are shown in the parentheses.

968 Extended Data Table 1 I Cryo-EM data collection, refinement and validation statistics

$969{ }^{*}$ For inactive mGlu2, different datasets were collected with different magnification of 970165,000 and 130,000 , corresponding to different pixel sizes of $0.82 \AA$ and $1.04 \AA$, 971 respectively.

972 'Particles picked from different datasets with different pixel sizes. The particles were then 973 combined using RELION 3.1.

$974{ }^{*}$ n.a., not applicable. The LocSpiral performs the local map enhancement based on 975 thresholding of amplitudes, which are obtained by using the spiral phase transformation. 976 The algorithm does not estimate or use the $B$ factor.

977 Extended Data Table 2 I Data collection and structure refinement statistics of mGlu2-

978 TMD-NAM563 and mGlu2-TMD-NAM597 complexes

979 *Numbers in parentheses refer to the highest-resolution shell.

980 Extended Data Table 3 I IP accumulation assays of wild-type (WT) and mutant mGlus 981 using a chimeric $\mathbf{G} \alpha$ protein $\mathbf{G} \alpha_{\text {qi9 }}$

982 The basal activity was calculated by subtracting the IP production measured in the control $983\left(\mathrm{G} \alpha_{\mathrm{qi} 9}\right)$ for the WT receptor and all the mutants, and is presented as percent of WT activity.

984 Data are shown as mean \pm SEM from at least three independent experiments. nd (not 985 determined) refers to data where a robust concentration response curve could not be 
986 established within the concentration range tested. ${ }^{* * *} P<0.001$ by one-way ANOVA followed

987 by Dunnett's post-test compared to the response of WT.

988 Sample size, the number of independent experiments performed in technical triplicate.

989 "Protein expression levels of mGlu2 and mGlu7 constructs at the cell surface were

990 determined in parallel by flow cytometry with an anti-Flag antibody (Sigma) and reported as

991 percent compared to the WT from at least three independent measurements performed in 992 duplicate.

993 The mutations were introduced in the wild-type receptor.

$994{ }^{\#}$ The span is defined as the window between the maximal agonist response $\left(E_{\max }\right)$ and the 995 vehicle (no agonist/PAM).

$996{ }^{\dagger} \mathrm{The} \mathrm{IC}_{50}$ ratio $\left(\mathrm{IC}_{50 \text { (mutant) }} / \mathrm{IC}_{50(\mathrm{WT})}\right)$ characterizes the effect of mutations on inhibitory 997 activity of NAMs.

$998+\mathrm{n}=29$. 


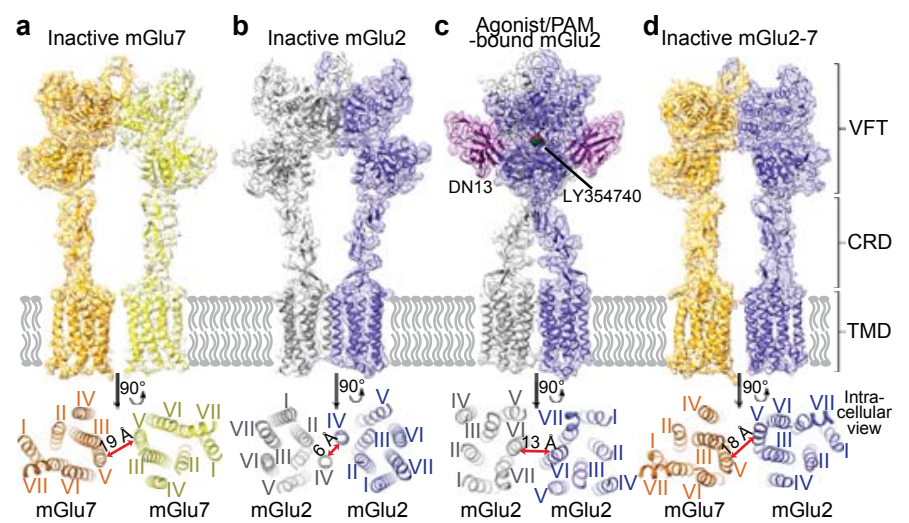




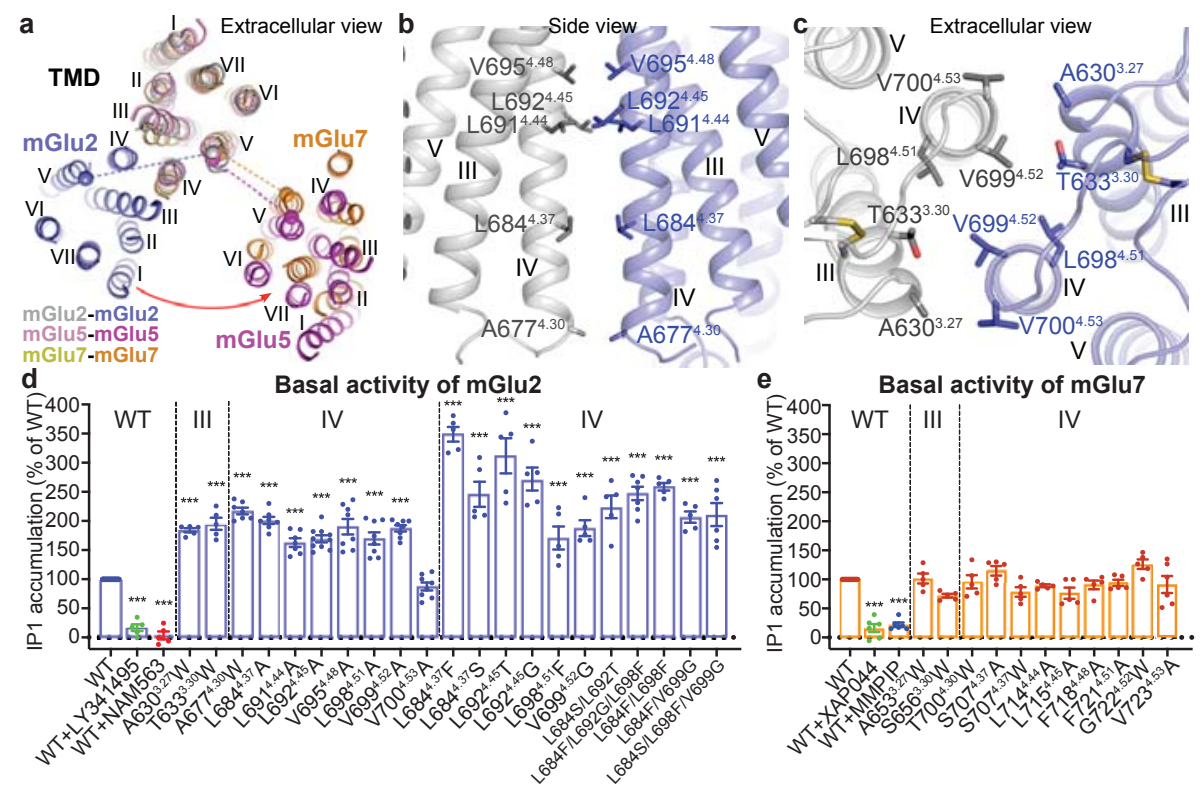




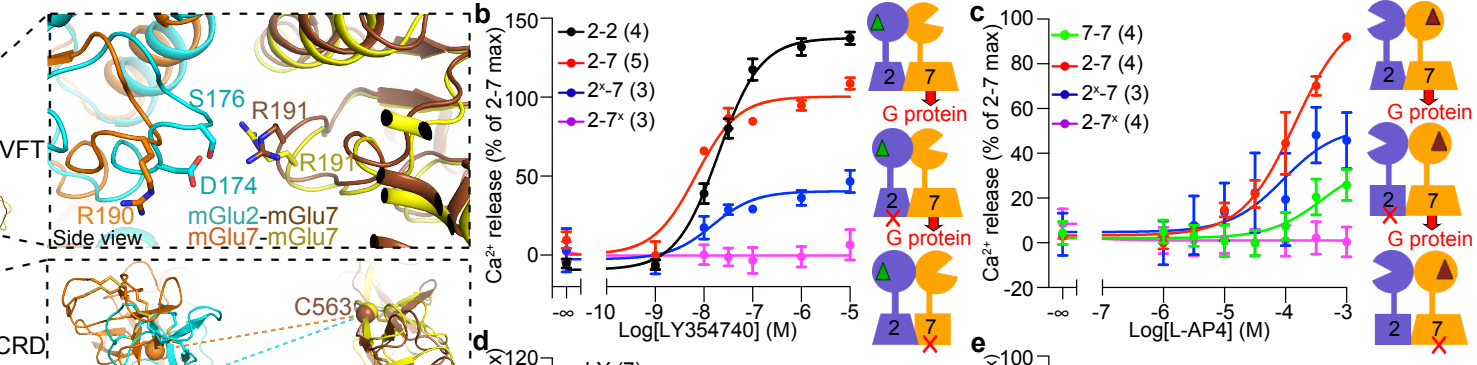

: 1 (2)

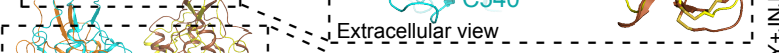

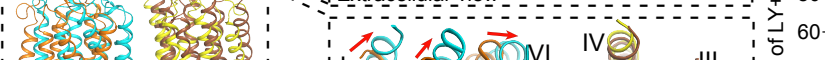

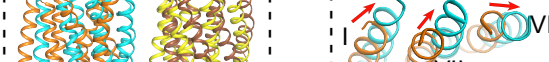

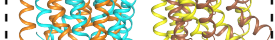

(8) 21 (III

L. $-2-2,2-2,-2$.

mGlu2-mGlu7 :

mGlu7-mGlu7

vo
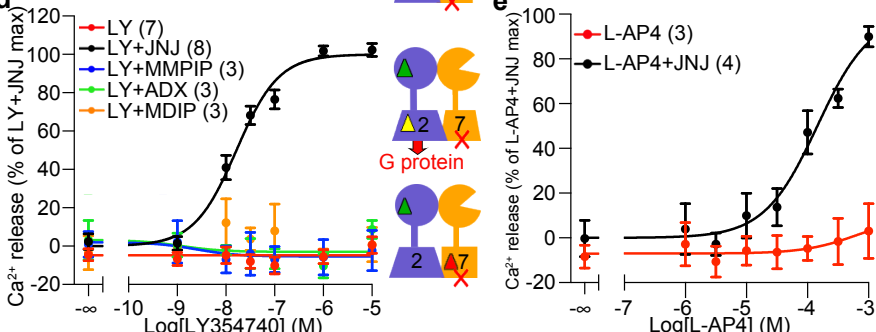

$\Delta 2$
protein
LY354

Q) 'Extracellular vieQ 